\title{
Relative hyperbolicity and relative quasiconvexity for countable groups
}

\author{
G CHRISTOPHER HRUSKA
}

\begin{abstract}
We lay the foundations for the study of relatively quasiconvex subgroups of relatively hyperbolic groups. These foundations require that we first work out a coherent theory of countable relatively hyperbolic groups (not necessarily finitely generated). We prove the equivalence of Gromov, Osin and Bowditch's definitions of relative hyperbolicity for countable groups.

We then give several equivalent definitions of relatively quasiconvex subgroups in terms of various natural geometries on a relatively hyperbolic group. We show that each relatively quasiconvex subgroup is itself relatively hyperbolic, and that the intersection of two relatively quasiconvex subgroups is again relatively quasiconvex. In the finitely generated case, we prove that every undistorted subgroup is relatively quasiconvex, and we compute the distortion of a finitely generated relatively quasiconvex subgroup.
\end{abstract}

20F65, 20F67

\section{Introduction}

Gromov introduced the theory of hyperbolic groups in [19]. In this theory, the quasiconvex subgroups play a central role. They are geometrically the simplest and most natural subgroups: those whose intrinsic geometry (the word metric with respect to a finite generating set) is preserved under the embedding into the hyperbolic group. The two most fundamental properties of quasiconvex subgroups of a hyperbolic group are the following.

(1) Each quasiconvex subgroup is itself word hyperbolic.

(2) The intersection of two quasiconvex subgroups is quasiconvex.

In the same article, Gromov also introduced relatively hyperbolic groups. In the present article, we lay the foundations for a theory of "relatively quasiconvex" subgroups, which are expected to play a central role in the theory of relatively hyperbolic groups. There are several equivalent ways to formulate this idea. Two of these formulations were 
introduced by Dahmani [11] and Osin [27] in special cases. Indeed Osin's definition has already been used in work of Martínez-Pedroza [26; 25] and Manning-MartínezPedroza [24].

Yet Dahmani's and Osin's definitions were not previously known to be equivalent, and neither of these notions was known to satisfy both of the fundamental properties above. In addition, no criterion was previously known for relative quasiconvexity using the "intrinsic" geometry of geodesics in the Cayley graph (for a finite generating set). In this article, we give several definitions of relative quasiconvexity, prove their equivalence, and use them to prove many properties of such subgroups. In particular, we show that relatively quasiconvex subgroups satisfy analogues of the two fundamental properties listed above.

\subsection{Non-finitely generated groups and relative hyperbolicity}

Historically the main conceptual difficulty to forming a satisfying theory of relative quasiconvexity has been the necessity of considering non-finitely generated groups. Relatively hyperbolic groups have been defined in different ways by Gromov [19], Farb [15] and Druţu-Sapir [13]. Three different model geometries arise in these three definitions. The geometry in Gromov's definition can be obtained by attaching "horoballs" to the peripheral subgroups. This definition generalizes the fundamental group of a finite volume hyperbolic manifold. The geometry in Farb's definition (of strong relative hyperbolicity) is obtained by collapsing each peripheral subgroup to a set of bounded diameter. This definition generalizes the structure of a free product acting on its Bass-Serre tree. The geometry in Drutu and Sapir's definition is the "intrinsic" geometry of the word metric with respect to a finite generating set. This definition generalizes the geometry of a CAT( 0$)$ space with isolated flats. These model geometries have substantially different flavors.

Farb's definition and Druţu and Sapir's definition each require that the group in question be finitely generated. However, Gromov's definition requires only that the group be countable. (It must act properly discontinuously on a proper metric space.) In the setting of finitely generated groups, these three definitions are equivalent. (See Bowditch [7] and Druțu-Sapir [13] for details.)

Bowditch [7] and Osin [27] have given variants of Farb's definition that do not require finitely generated groups. (They are variants in the sense that they essentially use the same model geometry introduced by Farb.) The exact relation between these definitions has not been clear. Many researchers have concluded that finite generation should be part of the definition of relative hyperbolicity. (Gromov and Osin are notable exceptions to this trend.) Indeed, no version of Druțu and Sapir's definition is known for non-finitely generated groups. 
Yet any natural definition of relatively quasiconvex subgroups will include non-finitely generated groups. Additionally, the intersection of two finitely generated relatively quasiconvex subgroups is often not finitely generated. Thus in order to formulate a natural theory of relative hyperbolicity, we must allow non-finitely generated relatively hyperbolic groups.

The following theorem reconciles the various existing definitions into a unified theory of relative hyperbolicity that includes all of the above examples. See Section 3 for precise statements of the various definitions of relative hyperbolicity.

Theorem 1.1 If $G$ is countable and $\mathbb{P}$ is a finite collection of infinite subgroups of $G$, then the definitions of relative hyperbolicity for $(G, \mathbb{P})$ given by Gromov, Bowditch and $O \sin$ are equivalent.

In fact, there are numerous examples of non-finitely generated relatively hyperbolic groups:

- A free product $A * B$ where $A$ or $B$ is not finitely generated is relatively hyperbolic with respect to the factors.

- A nonuniform lattice $\Gamma$ in a rank one Lie group $G$ over a nonarchimedian local field is relatively hyperbolic with respect to its parabolic subgroups by Lubotzky [23]. These lattices are never finitely generated. A typical example is the lattice $\Gamma=\mathrm{SL}_{2}\left(\mathbb{F}_{q}[t]\right)$ in the group $G=\mathrm{SL}_{2}\left(\mathbb{F}_{q}((1 / t))\right)$. In this case $\Gamma$ acts on the Bruhat-Tits tree for $G$ with quotient a ray.

- Many relatively quasiconvex subgroups of relatively hyperbolic groups are not finitely generated.

\subsection{Relative quasiconvexity}

As mentioned above, two different definitions of a relatively quasiconvex subgroup $H$ of a relatively hyperbolic group $G$ have been introduced by Dahmani and Osin in the special case that $G$ is finitely generated. Dahmani's "dynamical quasiconvexity" is defined in terms of the dynamics at infinity in Gromov's model geometry. Osin's "relative quasiconvexity" is defined in terms of Farb's "coned-off" model geometry and makes sense only for subgroups of a finitely generated relatively hyperbolic group. Dahmani's definition immediately implies that a dynamically quasiconvex subgroup is itself relatively hyperbolic. On the other hand, Martínez-Pedroza used Osin's definition to prove that the intersection of two relatively quasiconvex subgroups is again relatively quasiconvex [26]. Osin asked whether the two definitions are equivalent, and also 
asked whether all relatively quasiconvex subgroups are relatively hyperbolic (using his definitions) [27].

In this article, we clarify the notion of relatively quasiconvex subgroups by giving criteria for relative quasiconvexity in terms of the model geometries of Gromov, Farb and Druțu-Sapir. (Recall that Druţu and Sapir's geometry, the word metric for a finite generating set, is defined only when $G$ is finitely generated.) When $G$ is finitely generated, these subgroups coincide with those studied by Dahmani and Osin. A significant part of this paper involves showing that various definitions of relative quasiconvexity are equivalent. This equivalence has several consequences.

Theorem 1.2 Let $G$ be a countable group that is relatively hyperbolic with respect to a finite family of subgroups $\mathbb{P}=\left\{P_{1}, \ldots, P_{n}\right\}$.

(1) If $H \leq G$ is relatively quasiconvex, then $H$ is relatively hyperbolic with respect to a natural induced collection of subgroups.

(2) If $H_{1}, H_{2} \leq G$ are relatively quasiconvex, then $H_{1} \cap H_{2}$ is also relatively quasiconvex.

In the manifold setting, our characterization of relative quasiconvexity has the following corollary.

Corollary 1.3 Let $G$ be a geometrically finite subgroup of $\operatorname{Isom}(X)$ for $X$ a complete, simply connected manifold with pinched negative curvature. Then $H \leq G$ is relatively quasiconvex (with respect to the maximal parabolic subgroups of $G$ ) if and only if $H$ is geometrically finite.

Furthermore, if $H, K \leq G$ are geometrically finite then $H \cap K$ is also geometrically finite.

In the special case when $X$ is the real hyperbolic space $\mathbb{H}^{n}$, the second claim of the preceding corollary is due to Susskind-Swarup [31]. See also Corollary 1.6 below, which strengthens the first conclusion in the real hyperbolic case. The preceding corollary appears to be new even in the special case of complex hyperbolic manifolds.

If $G$ is relatively hyperbolic, $H \leq G$ is relatively quasiconvex, and both $H$ and $G$ are finitely generated, we compute the distortion of $H$ in $G$, which measures the difference between the word metric on $H$ and the word metric on $G$. See Theorem 10.5 for a more precise statement. 
Theorem 1.4 Let $G$ be relatively hyperbolic with respect to $P_{1}, \ldots, P_{n}$. Suppose $H \leq G$ is relatively quasiconvex, and both $H$ and $G$ are finitely generated. Then the distortion of $H$ in $G$ is a combination of the distortions of the infinite subgroups $O \leq P_{i}$ where $O=g \mathrm{Hg}^{-1} \cap P_{i}$.

More precisely, the distortion $\Delta_{H}^{G}$ of $H$ in $G$ satisfies

$$
f \preceq \Delta_{H}^{G} \preceq \bar{f}
$$

where $f$ is the supremum of the distortions of the subgroups $O \leq P_{i}$ and $\bar{f}$ is the superadditive closure of $f$.

The proof of this theorem uses a characterization of relative quasiconvexity in terms of the word metric on $G$ with respect to a finite generating set. Indeed Gromov's and Farb's model geometries are poorly suited to prove such a result, since the subgroups $P_{i}$ are badly distorted in these geometries (exponentially distorted in the Gromov model and crushed to a finite diameter in the Farb model). On the other hand, the proof is quite natural using the word metric because by Druț-Sapir [13] the peripheral subgroups $P_{i}$ are undistorted in $G$.

Druţu and Sapir previously showed that undistorted subgroups of a finitely generated relatively hyperbolic group are themselves relatively hyperbolic [13]. The following theorem places Drutu and Sapir's result in the context of relative quasiconvexity.

Theorem 1.5 (Undistorted $\Rightarrow$ relatively quasiconvex) Let $G$ be a finitely generated relatively hyperbolic group and let $H$ be a finitely generated subgroup. If $H$ is undistorted in $G$, then $H$ is relatively quasiconvex.

Using Theorem 1.4 and a result of the author from [22], we obtain a characterization of the geometrically finite subgroups $H$ of a geometrically finite Kleinian group $G$ that strengthens the conclusion of Corollary 1.3.

Corollary 1.6 Let $G$ be a geometrically finite subgroup of $\operatorname{Isom}\left(\mathbb{H}^{n}\right)$, and let $H \leq G$ be a subgroup. The following are equivalent.

(1) $H$ is geometrically finite.

(2) $H$ is relatively quasiconvex with respect to the maximal parabolic subgroups of $G$.

(3) $H$ is finitely generated and undistorted in $G$, in the sense that the inclusion $H \hookrightarrow G$ is a quasi-isometric embedding with respect to the word metrics for finite generating sets.

(4) $H$ is CAT( 0$)$-quasiconvex in $G$, in the sense that whenever $G$ acts properly, cocompactly and isometrically on any CAT( 0$)$ space $X$, the orbits of $H$ are quasiconvex in $X$. 


\subsection{Summary of the sections}

In Section 2 we review the definition of a horoball in a $\delta$-hyperbolic space and prove several lemmas about geodesics in horoballs. In Section 3 we explicitly state six different definitions of relative hyperbolicity. Section 4 is a review of Groves and Manning's construction of combinatorial horoballs based on a connected graph. We observe that their construction can be applied more generally to produce horoballs based on an arbitrary metric space. Using this observation, we state more general versions of some results of Groves and Manning that we apply in the following section. Section 5 consists of the proof that the six definitions of relative hyperbolicity are equivalent. Many of the directions are proved by observing that various proofs in the literature go through without change when one uses weaker hypotheses.

In Section 6 we introduce five equivalent definitions of relative quasiconvexity for subgroups of a relatively hyperbolic group. Section 7 contains a proof of the equivalence of the definitions introduced in the previous section. In Section 8 we turn our attention to the word metric on a relatively hyperbolic group $G$ with a finite generating set. In the case when $G$ is finitely generated, we characterize relatively quasiconvex subgroups $H$ in terms of the word metric on $G$.

In Section 9 we prove several basic results about relatively quasiconvex subgroups, including Theorems 1.2 and 1.5 and Corollary 1.3. We also characterize strongly relatively quasiconvex subgroups, which were introduced by Osin in [27]. Finally in Section 10 we examine distortions of relatively quasiconvex subgroups, proving Theorem 10.5 (Theorem 1.4) and Corollary 1.6.

\subsection{Remark}

Shortly after the initial circulation of this article, Agol, Groves and Manning [1] introduced another definition of a relatively quasiconvex subgroup. Manning and Martínez-Pedroza [24] have shown that this definition is equivalent to definition (QC-3) of the present article.

Acknowledgements The author wishes to thank Wen-yuan Yang for comments on an earlier version of this article. He is also grateful for the many helpful suggestions of the referee.

This research was supported by NSF grants DMS-0505659 and DMS-0731759. 


\section{Hyperbolic spaces and horoballs}

A geodesic space $(X, \rho)$ is $\delta$-hyperbolic if every geodesic triangle with vertices in $X$ is $\delta$-thin in the sense that each side lies in the $\delta$-neighborhood of the union of the other two sides. If $X$ is a $\delta$-hyperbolic space, the boundary (or boundary at infinity) of $X$, denoted $\partial X$, is the set of all equivalence classes of geodesic rays in $X$, where two rays $c, c^{\prime}$ are equivalent if the distance $\left.d\left(c(t), c^{\prime} t\right)\right)$ remains bounded as $t \rightarrow \infty$. The set $\partial X$ has a natural topology, which is compact and metrizable (see, for instance, Bridson-Haefliger [9] for details).

Increasing the constant $\delta$ if necessary, we will also assume that every geodesic triangle with vertices in $X \cup \partial X$ is $\delta$-thin.

Let $\Delta=\Delta(x, y, z)$ be a triangle with vertices $x, y, z \in X \cup \partial X$. A center of $\Delta$ is a point $w \in X$ such that the ball $B(w, \delta)$ intersects all three sides of the triangle. It is clear that each side of $\Delta$ contains a center of $\Delta$.

A function $h: X \rightarrow \mathbb{R}$ is a horofunction about a point $\xi \in \partial X$ if there is a constant $D_{0}$ such that the following holds. Let $\Delta(x, y, \xi)$ be a geodesic triangle, and let $w \in X$ be a center of the triangle. Then

$$
|(h(x)+\rho(x, w))-(h(y)-\rho(y, w))|<D_{0} .
$$

A closed subset $B \subseteq X$ is a horoball centered at $\xi$ if there is a horofunction $h$ about $\xi$ and a constant $D_{1}$ such that $h(x) \geq-D_{1}$ for all $x \in B$ and $h(x) \leq D_{1}$ for all $x \in X-B$.

We remark that every horoball or horofunction has a unique center, and every $\xi \in \partial X$ is the center of a horofunction (see Gromov [19, Section 7.5] for details).

Lemma 2.1 Let $B$ be a horoball of a $\delta$-hyperbolic space $(X, \rho)$. For each $L>0$, there is a constant $M_{0}=M_{0}(B, L)$ such that any geodesic $c$ in $\mathcal{N}_{L}(X-B) \cap B$ has length at most $M$.

The notation $\mathcal{N}_{r}(A)$ denotes the open $r$-neighborhood of $A$; ie, the set of all points at a distance less than $r$ from $A$.

Proof Let $\xi \in \partial X$ be the center of $B$, and choose a horofunction $h$ and constants $D_{0}, D_{1}$ for $B$ as above. Suppose $c$ has endpoints $x_{0}$ and $x_{1}$, and choose geodesic rays $\left[x_{i}, \xi\right)$ for $i=1,2$. Choose $z \in c$ within a distance $2 \delta$ of both rays $\left[x_{i}, \xi\right)$, and choose a ray $[z, \xi)$. 
In order to complete the proof, it suffices to bound $\rho\left(x_{i}, z\right)$ from above. We first compute an upper bound for $h(z)$. If we choose $w \in X-B$ such that $\rho(w, z)<L$, then $h(w) \leq D_{1}$. Applying the definition of horofunction to a triangle with vertices $w, z, \xi$ and center $p \in[w, z]$, gives

$$
h(z)+\rho(z, p)<h(w)+\rho(z, p)+D_{0}
$$

Thus we obtain the following upper bound:

$$
\begin{aligned}
h(z) & <h(w)+\rho(w, p)-\rho(z, p)+D_{0} \\
& \leq h(w)+\rho(w, z)+D_{0} \\
& \leq D_{1}+L+D_{0} .
\end{aligned}
$$

On the other hand, since $x_{i} \in B$ we have a lower bound $h\left(x_{i}\right) \geq-D_{1}$.

Let $\Delta_{i}=\Delta\left(x_{i}, z, \xi\right)$ be the triangle with sides $\left[x_{i}, \xi\right),[z, \xi)$, and $\left[x_{i}, z\right]$, where $\left[x_{i}, z\right]$ is the portion of $c$ from $x_{i}$ to $z$. Observe that $z$ is a center of $\Delta_{i}$. Applying the definition of horofunction to $\Delta_{i}$, we have

$$
\rho\left(x_{i}, z\right)<h(z)-h\left(x_{i}\right)+D_{0} \leq\left(D_{1}+L+D_{0}\right)+D_{1}+D_{0},
$$

completing the proof.

The following result can be also proved by a similar proof.

Lemma 2.2 Let $B$ and $B^{\prime}$ be horoballs of $(X, \rho)$ centered at the same point $\xi \in \partial X$. There is a constant $M_{1}=M_{1}\left(B, B^{\prime}\right)$ such that any geodesic $c$ in $B-B^{\prime}$ has length at most $M_{1}$.

Lemma 2.3 Let $B$ be a horoball of $(X, \rho)$ with corresponding horofunction $h$. There is a constant $M_{2}=M_{2}(B, h)$ such that the following holds. Suppose $x, y \in X-B$ satisfy $h(x)=h(y)=0$. Then for any geodesic $c$ joining $x$ and $y$ we have

$$
c \cap(X-B) \subseteq \mathcal{N}_{M_{2}}(\{x, y\})
$$

Proof Let $z$ be an arbitrary point of $c \cap(X-B)$. Then $h(z) \leq D_{1}$. Choose rays $[x, \xi),[y, \xi)$ and $[z, \xi)$. The point $z$ is within a distance $2 \delta$ of one of the rays $[x, \xi)$ or $[y, \xi)$, say $[x, \xi)$. Since $z$ is a center of $\Delta=\Delta(x, z, \xi)$, the definition of horofunction implies that

$$
\rho(x, z) \leq h(z)+D_{0} \leq D_{1}+D_{0}
$$

completing the proof. 


\section{Notions of relative hyperbolicity for countable groups}

It is well-known that finitely generated relatively hyperbolic groups can be characterized in several equivalent ways. In this section, we discuss natural extensions of these properties to the setting of countable groups. Throughout the section, $G$ is a countable group with a finite collection of subgroups $\mathbb{P}=\left\{P_{1}, \ldots, P_{n}\right\}$.

\subsection{Geometrically finite groups}

The first definition of relative hyperbolicity is in terms of dynamical properties of an action on a compact space $M$. More precisely, below we define a group $G$ to be relatively hyperbolic if it admits a geometrically finite convergence group action on some compactum $M$. In fact by Corollary 5.3 below the compactum $M$ always arises as the boundary of a $\delta$-hyperbolic space on which $G$ acts. Thus we lose no generality by keeping this geometric example in mind throughout the following definitions.

The notion of a convergence group action was introduced by Gehring and Martin in [18] to axiomatize certain dynamical properties of the action of a Kleinian group on its limit set in the ideal sphere at infinity of real hyperbolic space. The dynamical version of geometrical finiteness defined here was introduced by Beardon and Maskit [3] for Kleinian groups.

A convergence group action is an action of a group $G$ on a compact, metrizable space $M$ satisfying the following conditions, depending on the cardinality of $M$ :

- If $M$ is the empty set, then $G$ is finite.

- If $M$ has exactly one point, then $G$ can be any countable group.

- If $M$ has exactly two points, then $G$ is virtually cyclic.

- If $M$ has at least three points, then the action of $G$ on the space of distinct (unordered) triples of points of $M$ is properly discontinuous.

In the first three cases the action is elementary, and in the final case the action is nonelementary.

Suppose $G$ has a convergence group action on $M$. An element $g \in G$ is loxodromic if it has infinite order and fixes exactly two points of $M$. A subgroup $P \leq G$ is a parabolic subgroup if it is infinite and contains no loxodromic element. A parabolic subgroup $P$ has a unique fixed point in $M$, called a parabolic point. The stabilizer of a parabolic point is always a maximal parabolic group. A parabolic point $p$ with stabilizer $P:=\operatorname{Stab}_{G}(p)$ is bounded if $P$ acts cocompactly on $M-\{p\}$. A point 
$\xi \in M$ is a conical limit point if there exists a sequence $\left(g_{i}\right)$ in $G$ and distinct points $\zeta_{0}, \zeta_{1} \in M$ such that $g_{i}(\xi) \rightarrow \zeta_{0}$, while for all $\eta \in M-\{\xi\}$ we have $g_{i}(\eta) \rightarrow \zeta_{1}$.

Tukia has shown that every properly discontinuous action of a group $G$ on a proper $\delta$-hyperbolic space induces a convergence group action on the boundary at infinity [34] (a similar result was proved independently by Freden [16]).

A convergence group action of $G$ on $M$ is geometrically finite if every point of $M$ is either a conical limit point or a bounded parabolic point. In addition if $\mathbb{P}$ is a set of representatives of the conjugacy classes of maximal parabolic subgroups, then we say that the action of the pair $(G, \mathbb{P})$ on $M$ is geometrically finite. Observe that every elementary convergence group action is geometrically finite.

The following definition was proposed by Bowditch in [7] and studied by Yaman in [36] (with the additional assumption that the peripheral subgroups $P \in \mathbb{P}$ are finitely generated).

Definition 3.1 $(\mathrm{RH}-1)$ Suppose $(G, \mathbb{P})$ has a geometrically finite convergence group action on a compact, metrizable space $M$. Then $(G, \mathbb{P})$ is relatively hyperbolic.

The following definition is similar to the preceding one, except that we assume that the compact space $M$ is the boundary of a $\delta$-hyperbolic space. This definition was introduced by Bowditch in [7].

Definition 3.2 (RH-2) Suppose $G$ has a properly discontinuous action on a proper $\delta$-hyperbolic space $X$ such that the induced convergence group action on $\partial X$ is geometrically finite. If $\mathbb{P}$ is a set of representatives of the conjugacy classes of maximal parabolic subgroups then $(G, \mathbb{P})$ is relatively hyperbolic.

In this case, we also say that $(G, \mathbb{P})$ acts geometrically finitely on $X$.

\subsection{Cusp uniform actions}

The next definition is essentially Gromov's original definition of relative hyperbolicity from [19], as stated by Bowditch in [7]. The structure is analogous to the well-known decomposition of a finite volume hyperbolic manifold as the union of a compact part together with finitely many cusps due to Garland-Raghunathan [17] (see also Thurston [33, Section 4.5]).

Definition 3.3 (RH-3) Suppose $G$ acts properly discontinuously on a proper $\delta-$ hyperbolic space $X$, and $\mathbb{P}$ is a set of representatives of the conjugacy classes of maximal parabolic subgroups. Suppose also that there is a $G$-equivariant collection of 
disjoint horoballs centered at the parabolic points of $G$, with union $U$ open in $X$, such that the quotient of $X-U$ by the action of $G$ is compact. Then $(G, \mathbb{P})$ is relatively hyperbolic.

In this case, we say that the action of $(G, \mathbb{P})$ on $X$ is cusp uniform, and the space $Y=X-U$ is a truncated space for the action. By a slight abuse of notation, we refer to the horoballs of $U$ as horoballs of $Y$. If $U^{\prime}$ is any other $G$-equivariant family of disjoint open horoballs centered at the parabolic points of $X$, then $G$ also acts cocompactly on $Y^{\prime}=X-U^{\prime}$, and hence $Y^{\prime}$ is also a truncated space for the cusp uniform action of $(G, \mathbb{P})$ on $X$ (see Bowditch [7] for details).

\subsection{Fine hyperbolic graphs}

The following definition of relative hyperbolicity was proposed by Bowditch in [7] as an abstraction of the Farb approach that does not require finite generation. Bowditch studied finitely generated groups satisfying this condition, but it is a completely trivial matter to remove the finite generation hypothesis from his definition.

A graph $K$ is fine if each edge of $K$ is contained in only finitely many circuits of length $n$ for each $n$.

Definition 3.4 (RH-4) Suppose $G$ acts on a $\delta$-hyperbolic graph $K$ with finite edge stabilizers and finitely many orbits of edges. If $K$ is fine, and $\mathbb{P}$ is a set of representatives of the conjugacy classes of infinite vertex stabilizers then $(G, \mathbb{P})$ is relatively hyperbolic.

\subsection{The coned-off Cayley graph and Bounded Coset Penetration}

Next we consider Farb's notion of (strong) relative hyperbolicity from [15]. (Our terminology here does not agree with Farb's and has been adjusted to be more consistent with the other definitions of relative hyperbolicity in this article.)

In brief, Farb's definition requires that the "coned-off" Cayley graph is hyperbolic and satisfies Bounded Coset Penetration (defined below). Farb also considered a weak version of relative hyperbolicity, which has many interesting applications. We will not discuss weak relative hyperbolicity here.

Farb originally proposed this definition for finitely generated groups. After the work of Bowditch and Osin [7;27], it is clear that the following condition is the natural formulation of Farb's notion in the non-finitely generated setting.

Let $G$ be a group with a collection of subgroups $\mathbb{P}=\left\{P_{1}, \ldots, P_{n}\right\}$. A set $\mathcal{S}$ is a relative generating set for the pair $(G, \mathbb{P})$ if the set $\mathcal{S} \cup P_{1} \cup \cdots \cup P_{n}$ is a generating set 
for $G$ in the traditional sense. We will always implicitly assume that $\mathcal{S}$ is symmetrized, so that $\mathcal{S}=\mathcal{S}^{-1}$. Let $\Gamma$ be the Cayley graph Cayley $(G, \mathcal{S})$. Note that $\Gamma$ is connected if and only if $\mathcal{S}$ is a traditional generating set for $G$. We do not require connectedness of $\Gamma$.

Form a new graph $\hat{\Gamma}(G, \mathbb{P}, \mathcal{S})$, called the coned-off Cayley graph, as follows. For each left coset $g P$ with $g \in G$ and $P \in \mathbb{P}$, add a new vertex $v(g P)$ to $\Gamma$, and add an edge of length $1 / 2$ from this new vertex to each element of $g P$. The key point to note here is that the coned-off Cayley graph is connected if and only if $\mathcal{S}$ is a relative generating set for $(G, \mathbb{P})$.

Given an oriented path $\gamma$ in the coned-off Cayley graph, we say that $\gamma$ penetrates the coset $g P$ if $\gamma$ passes through the cone point $v(g P)$. A vertex $v_{i}$ of $p$ immediately preceding the cone point is an entering vertex of $p$ in the coset $g P$. Exiting vertices are defined similarly. Observe that entering and exiting vertices are always elements of $G$, and hence can also be considered as vertices of $\Gamma$. A path $\gamma$ in the coned-off Cayley graph is without backtracking if, for every coset $g P$ which $\gamma$ penetrates, $\gamma$ never returns to $g P$ after leaving $g P$.

Definition 3.5 (Bounded Coset Penetration) Let $\mathcal{S}$ be a relative generating set for $(G, \mathbb{P})$. The triple $(G, \mathbb{P}, \mathcal{S})$ satisfies Bounded Coset Penetration if for each $\lambda \geq 1$, there is a constant $a(\lambda)>0$ such that if $\gamma$ and $\gamma^{\prime}$ are $(\lambda, 0)$-quasigeodesics without backtracking in the coned-off Cayley graph $\hat{\Gamma}(G, \mathbb{P}, \mathcal{S})$ with initial endpoints $\gamma_{-}=\gamma_{-}^{\prime}$ and terminal endpoints $\gamma_{+}$and $\gamma_{+}^{\prime}$, and $d_{\mathcal{S}}\left(\gamma_{+}, \gamma_{+}^{\prime}\right) \leq 1$, then the following two conditions hold.

(1) If $\gamma$ penetrates a coset $g P$, but $\gamma^{\prime}$ does not penetrate $g P$, then the entering vertex and exiting vertex of $\gamma$ in $g P$ are at an $\mathcal{S}$-distance at most $a$ from each other.

(2) If $\gamma$ and $\gamma^{\prime}$ both penetrate a coset $g P$, then the entering vertices of $\gamma$ and $\gamma^{\prime}$ in $g P$ are at an $\mathcal{S}$-distance at most $a$ from each other. Similarly, the exiting vertices are at an $\mathcal{S}$-distance at most $a$ from each other.

Definition 3.6 (RH-5) Suppose $G$ is finitely generated relative to $\mathbb{P}$, and each $P_{i}$ is infinite. The pair $(G, \mathbb{P})$ is relatively hyperbolic if for some (every) finite relative generating set $\mathcal{S}$, the coned-off Cayley graph $\hat{\Gamma}(G, \mathbb{P}, \mathcal{S})$ is $\delta$-hyperbolic and $(G, \mathbb{P}, \mathcal{S})$ has Bounded Coset Penetration.

In the finitely generated case, $\mathrm{BCP}$ is independent of the choice of generating set due to the following fact: a change of generating set induces a quasi-isometry of Cayley 
graphs and also a quasi-isometry of coned-off Cayley graphs. However, in general this independence is less obvious. The difficulty is that a Cayley graph for a nongenerating set is not connected. It is possible for two group elements to be connected by an edge in one Cayley graph and lie in distinct components of another. In Section 5 we indicate a proof of this independence based on arguments of Dahmani [12].

\subsection{Linear relative Dehn function}

Finally we state the definition due to Osin in terms of relative isoperimetric functions introduced in [27]. The definition, as presented here, includes only countable groups $G$ with finitely many infinite peripheral subgroups $\mathbb{P}=\left\{P_{1}, \ldots, P_{n}\right\}$. We remark that Osin's theory does not impose such restrictions. However, these restrictions are necessary for the equivalence with other definitions of relative hyperbolicity.

Let $G$ be a group with a collection of subgroups $\mathbb{P}$. If $(G, \mathbb{P})$ has a relative generating set $\mathcal{S}$, then there is a canonical homomorphism from the group

$$
K:=F(\mathcal{S}) *(* P \in \mathbb{P} \widetilde{P})
$$

onto $G$. Here $F(\mathcal{S})$ denotes the group freely generated by $\mathcal{S}$, and $\widetilde{P}$ denotes an abstract group isomorphic to $P$. Let $N$ be the kernel of this homomorphism.

If $\mathcal{R} \subseteq N$ is a subset whose normal closure in $K$ is equal to $N$, then we say that $G$ has a relative presentation

$$
\langle\mathbb{P}, \mathcal{S} \mid \mathcal{R}\rangle \text {. }
$$

If $\mathcal{S}$ and $\mathcal{R}$ are both finite, then the relative presentation above is a finite relative presentation.

Suppose $(G, \mathbb{P})$ has a relative presentation as above. Consider the disjoint union

$$
\mathcal{P}:=\coprod_{P \in \mathbb{P}}(\widetilde{P}-\{1\})
$$

Each word $W$ in the alphabet $(\mathcal{S} \cup \mathcal{P})$ naturally represents both an element of $K$ and an element of $G$ (via the quotient map above). If $W$ represents 1 in $G$, then in $K$ we have an equation

$$
W=\prod_{K} \prod_{i=1}^{\ell} f_{i}^{-1} R_{i} f_{i}
$$

where $R_{i} \in \mathcal{R}$ and $f_{i} \in K$ for each $i$.

A function $f: \mathbb{N} \rightarrow \mathbb{N}$ is a relative isoperimetric function for the relative presentation $\langle\mathbb{P}, \mathcal{S} \mid \mathcal{R}\rangle$ if for each $m \in \mathbb{N}$ and any word $W$ over $(\mathcal{S} \cup \mathcal{P})$ of length at most $m$ 
representing the identity of $G$, we have an equation in $K$ of the form $(\dagger)$ with $\ell \leq f(m)$. We remark that some relative presentations do not admit a finite relative isoperimetric function. The relative Dehn function of a relative presentation is the smallest possible relative isoperimetric function. If there does not exist a relative isoperimetric function with finite values, we say that the relative Dehn function is not well-defined.

Definition 3.7 (RH-6) $\quad$ Suppose $\mathbb{P}$ is a finite collection of infinite subgroups of a countable group $G$. If $(G, \mathbb{P})$ has a finite relative presentation, and the relative Dehn function is well-defined and linear for some/every finite relative presentation then $(G, \mathbb{P})$ is relatively hyperbolic.

We remark that if one finite relative presentation has a linear relative Dehn function then so does any other by Osin [27, Theorem 2.34].

\section{The cusped space}

Our immediate goal is to prove that the definitions of relative hyperbolicity for countable groups given above are equivalent. As discussed below in Section 5 several of the necessary implications are either already known or follow from straightforward modifications of existing proofs in the literature.

In this section we lay the groundwork for the implication (RH-6) $\Rightarrow(\mathrm{RH}-3)$. The main step is the construction of a space obtained by attaching certain "combinatorial horoballs" to $G$ along the left cosets of the peripheral subgroups. The idea of gluing horoballs onto a finitely generated group is due to Cannon-Cooper [10], and variations have been studied by Bowditch [7], Rebbechi [29] and Groves-Manning [20].

The construction presented here is closely modeled on Groves and Manning's combinatorial horoballs. Groves and Manning constructed horoballs based on an arbitrary connected graph, and showed that such a horoball is always $\delta$-hyperbolic. A minor modification of the Groves-Manning construction produces connected horoballs based on any metric space. If the base metric space is discrete and proper, the resulting horoball is a locally finite $\delta$-hyperbolic graph.

Using this modification, Groves and Manning's proofs about finitely generated relatively hyperbolic groups extend to any countable relatively hyperbolic group equipped with a proper, left invariant metric.

Definition 4.1 (Combinatorial horoballs) Let $(P, d)$ be a metric space. The combinatorial horoball based on $P$, denoted $\mathcal{H}(P, d)$ is the graph defined as follows: 
(1) $\mathcal{H}^{(0)}=P \times \mathbb{N}$.

(2) $\mathcal{H}^{(1)}$ contains the following two types of edges:

(a) For each $k \in \mathbb{N}$ and $p, q \in P$, if $0<d(p, q) \leq 2^{k}$ then there is a horizontal edge connecting $(p, k)$ and $(q, k)$.

(b) For each $k \in \mathbb{N}$ and $p \in P$, there is a vertical edge connecting $(p, k)$ and $(p, k+1)$.

As in Groves-Manning [20], we consider each edge of $\mathcal{H}$ to have length one, and we endow $\mathcal{H}$ with the induced length metric $d_{\mathcal{H}}$. We identify $P$ with its image $P \times\{0\}$ in $\mathcal{H}$.

The following result is proved by Groves and Manning under the additional hypothesis that $(P, d)$ is the set of vertices of a connected metric graph where each edge has length one [20, Section 3.1]. The proof given there extends with no changes to the current setting.

Theorem 4.2 Let $(P, d)$ be any metric space (with all distances finite). Then $\mathcal{H}(P, d)$ is connected and $\delta$-hyperbolic for some $\delta \geq 0$ independent of $(P, d)$. Moreover, if $(P, d)$ is a discrete, proper metric space, then $\mathcal{H}(P, d)$ is a locally finite graph.

Definition 4.3 (The augmented space) Suppose $G$ is a countable group with subgroups $\mathbb{P}=\left\{P_{1}, \ldots, P_{n}\right\}$. Let $\mathcal{S}$ be a finite relative generating set for $(G, \mathbb{P})$, and choose a proper, left invariant metric $d_{i}$ on each $P_{i}$. The metric $d_{i}$ induces a metric (again called $d_{i}$ ) on each left coset $g P_{i}$ using left translation by $g$. The metric space $\left(g P_{i}, d_{i}\right)$ is locally finite. Consequently, $\mathcal{H}\left(g P_{i}, d_{i}\right)$ is a locally finite connected graph.

Let $\Gamma$ be the graph Cayley $(G, \mathcal{S})$. The augmented space is the space

$$
X:=\Gamma \cup\left(\bigcup \mathcal{H}\left(g P_{i}, d_{i}\right)^{(1)}\right),
$$

where the inner union ranges over all $i=1, \ldots, n$ and all left cosets $g P_{i}$ of $P_{i}$, and where each coset $g P_{i} \subseteq \Gamma^{(0)}$ is identified with $g P_{i} \subset \mathcal{H}\left(g P_{i}, d_{i}\right)$ in the obvious way.

Observe that $X$ is locally finite since each combinatorial horoball is locally finite and the collection of cosets $g P_{i}$ is locally finite in $G$ (ie, each finite subset of $G$ intersects only finitely many such cosets). Furthermore $X$ is connected because $\mathcal{S}$ is a relative generating set. The natural action of $G$ on $X$ is properly discontinuous and isometric.

The next theorem follows from arguments of Groves-Manning in [20, Section 3.3]. Groves and Manning assume that $G$ is finitely generated, but their proofs remain valid when $G$ is countable and $X$ is constructed as above. The only difference here is that 
we have imposed a proper, left invariant metric on each peripheral subgroup, while Groves and Manning metrize each peripheral subgroup using the word metric for a finite generating set.

Theorem 4.4 Suppose $G$ is countable and $\mathbb{P}$ is a finite collection of subgroups of $G$. Choose a finite relative generating set $\mathcal{S}$ for $(G, \mathbb{P})$. For each $P_{i} \in \mathbb{P}$ choose a proper, left invariant metric $d_{i}$. If $(G, \mathbb{P})$ has a finite relative presentation with a linear relative Dehn function, then the augmented space $X$ is $\delta$-hyperbolic.

\section{Equivalence of notions of relative hyperbolicity for count- able groups}

The goal of this section is the following theorem.

Theorem 5.1 The six definitions of relative hyperbolicity given above are equivalent. In addition, condition ( $\mathrm{RH}-5)$ does not depend on the choice of relative generating set.

Our strategy for proving the theorem is to explain the following implications:

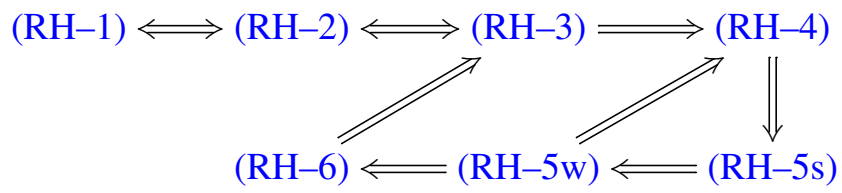

where (RH-5s) denotes the strong condition that (RH-5) holds for every finite relative generating set, and (RH-5w) denotes the weak condition that it holds for some particular finite relative generating set. We have included the implication ( $\mathrm{RH}-5 \mathrm{w}) \Rightarrow(\mathrm{RH}-4)$ in order to give a more direct proof of the equivalence of the weak and strong forms of (RH-5).

In fact most of the ingredients already exist in the literature. Some are proved completely, and others are proved for the finitely generated case but the proofs don't really use finite generation. In several places we replace the Cayley graph for a finite generating set with the (possibly disconnected) Cayley graph for a finite relative generating set. With this small change of perspective, the proofs in the literature go through almost verbatim.

As explained in the previous section, the most significant changes occur in our proof of (RH-6) $\Rightarrow$ (RH-3), where we need to modify the Groves-Manning construction of combinatorial horoballs to account for non-finitely generated groups. Note that we also use combinatorial horoballs to prove $(\mathrm{RH}-1) \Rightarrow(\mathrm{RH}-2)$ in the elementary parabolic case. 
The first implication we discuss is a consequence of the following theorem due to Yaman.

Theorem 5.2 [36] If $G$ acts as a nonelementary geometrically finite convergence group on a metrizable compactum $M$, then there is a proper $\delta$-hyperbolic space $X$ on which $G$ acts properly such that $M$ is $G$-equivariantly homeomorphic to $\partial X$ with its induced $G$-action.

Corollary 5.3 Definitions ( $\mathrm{RH}-1)$ and (RH-2) are equivalent.

Proof The implication $(\mathrm{RH}-2) \Rightarrow(\mathrm{RH}-1)$ is obvious. Now suppose $(G, \mathbb{P})$ satisfies (RH-1). If the action of $G$ on $M$ is nonelementary, then we are done by Theorem 5.2.

If the action on $M$ is elementary, it is a simple matter to construct an appropriate hyperbolic space $X$ as follows. If $M$ is empty, then $G$ acts trivially on a point $X=\{a\}$. If $M$ has one point then we choose a proper, left invariant metric $d$ on $G$, and let $G$ act properly on the combinatorial horoball $X=\mathcal{H}(G, d)$. If $M$ has two points, then $G$ is virtually cyclic and acts properly on the line $X=\mathbb{R}$. In this last case, arrange the action so that $g \in G$ preserves the orientation of $\mathbb{R}$ if and only if $g$ fixes both points of $M$. In each case it is clear that the induced action on $\partial X$ is equivalent to the given action on $M$, establishing (RH-2).

The equivalence (RH-2) $\Leftrightarrow(\mathrm{RH}-3)$ was discovered by Thurston in the classical setting of 3-dimensional Kleinian groups [32], and has been extended to various manifold cases by Apanasov [2] and Bowditch [4; 5]. The following result due to Bowditch [7] extends this equivalence to actions on $\delta$-hyperbolic spaces.

Theorem 5.4 [7] Let $G$ act properly on a proper $\delta$-hyperbolic space $X$. Let $\mathbb{P}$ be a finite family of subgroups of $G$. Then the action of $(G, \mathbb{P})$ on $X$ is geometrically finite if and only if it is cusp uniform.

Corollary 5.5 Definitions (RH-2) and (RH-3) are equivalent.

The following is one of the main theorems of [7]. We note that the result is stated with the extra assumption that the peripheral subgroups are finitely generated, but this hypothesis plays no role in the proof and is present only to make the result consistent with Bowditch's convention that peripheral subgroups should be finitely generated.

Theorem 5.6 [7] Definition (RH-3) implies (RH-4). 
As an aside, we remark that Bowditch also proves the converse ( $\mathrm{RH}-4) \Rightarrow(\mathrm{RH}-3)$ for finitely generated groups. However, his proof uses the finite generation hypothesis in an essential manner, and the proof does not extend to non-finitely generated groups. This implication is not necessary for our purposes.

Recall that in the finitely generated case $(\mathrm{RH}-5 \mathrm{~s})$ and $(\mathrm{RH}-5 \mathrm{w})$ are equivalent by a standard argument. This standard argument does not extend in the obvious way to the non-finitely generated case.

Bowditch claims without proof in [7] that (RH-4) and (RH-5) are equivalent for finitely generated groups. Dahmani provides a sketch in [11] and a complete proof in an appendix to his thesis [12]. Dahmani's proof extends with trivial modifications to the general case. For the benefit of the reader, we sketch the outline of a proof based on Dahmani's arguments indicating places where modifications must be made.

Proof of $(\mathbf{R H}-4) \Rightarrow(\mathbf{R H}-5 \mathbf{s}) \quad$ Suppose $G$ acts on a fine, $\delta$-hyperbolic graph $K$ with finite edge stabilizers and finitely many orbits of edges. Choose edges representing the $G$-orbits, and let $\left\{v_{1}, \ldots, v_{\ell}\right\}$ be the set of all vertices incident to any of these finitely many edges. If $v_{i}$ and $v_{j}$ lie in the same $G$-orbit, choose $g_{i j} \in G$ so that $g_{i j}\left(v_{i}\right)=v_{j}$. Then $G$ is generated by the stabilizers of the $v_{i}$ and the finitely many elements $g_{i j}$ (see, for instance, Bridson-Haefliger [9, Theorem I.8.10]). In particular, $G$ is finitely generated with respect to the infinite vertex stabilizers.

Now let $\mathcal{S}$ be an arbitrary finite relative generating set for $(G, \mathbb{P})$. Observe that the coned-off Cayley graph $\widehat{\Gamma}:=\widehat{\Gamma}(G, \mathbb{P}, \mathcal{S})$ has trivial edge stabilizers and finitely many orbits of edges. The argument in [12, Lemma A.4] produces a fine graph $K^{\prime}$ with finite edge stabilizers and finitely many orbits of edges such that $K$ and $\widehat{\Gamma}$ both embed equivariantly and simplicially into $K^{\prime}$. Any subgraph of a fine graph is fine, so $\widehat{\Gamma}$ is fine. Furthermore, a connected, equivariant subgraph of $K^{\prime}$ is quasi-isometric to $K^{\prime}$. Since $K$ is $\delta$-hyperbolic, it follows that $\widehat{\Gamma}$ is quasi-isometric to $K$, so $\widehat{\Gamma}$ is $\delta^{\prime}$-hyperbolic for some $\delta^{\prime} \geq 0$. Now by [12, Lemma A.5], the fineness of the coned-off Cayley graph $\hat{\Gamma}$ implies that $(G, \mathbb{P}, \mathcal{S})$ has Bounded Coset Penetration. (The proof of [12, Lemma A.5] never uses the connectedness of $\Gamma$, so it remains valid when $\mathcal{S}$ is a relative generating set, as opposed to a generating set in the traditional sense.)

Proof of $(\mathbf{R H}-5 \mathbf{w}) \Rightarrow(\mathbf{R H}-4)$ This implication follows directly from [12, Proposition A.1] with no change, as Dahmani's proof does not use the finite generation hypothesis.

Proof of $(\mathbf{R H}-\mathbf{5 w}) \Rightarrow$ (RH-6) This implication is proved by Osin in the finitely generated case in an appendix to [27]. However, he never uses the finite generation 
hypothesis in the proof. Again, the finite generation hypothesis seems to be present only to make the statement consistent with Farb's original version of ( $\mathrm{RH}-5)$ for finitely generated groups.

We remark that Osin also gives a substantially more involved proof of the converse $(\mathrm{RH}-6) \Rightarrow(\mathrm{RH}-5)$ for finitely generated groups. These arguments use finite generation extensively, and it is not clear whether the same arguments go through in general. Note that we do not require this implication.

Proof of (RH-6) $\Rightarrow$ (RH-3) Choose any finite relative generating set $\mathcal{S}$ for $(G, \mathbb{P})$ and choose a proper, left invariant metric $d_{i}$ for each subgroup $P_{i} \in \mathbb{P}$. By Theorem 4.4, the augmented space $X$ corresponding to these data is connected and $\delta$-hyperbolic for some $\delta \geq 0$. It is straightforward to verify that the combinatorial horoballs of $X$ are also horoballs as defined in Section 2. Deleting these horoballs from $X$ gives the (possibly disconnected) Cayley graph $\operatorname{Cayley}(G, \mathcal{S})$ for $G$, on which $G$ acts with compact quotient. Therefore $(G, \mathbb{P})$ is relatively hyperbolic in the sense of (RH-3).

\section{Relatively quasiconvex subgroups: Definitions}

Now that we have established the notion of relative hyperbolicity for countable groups, we turn our attention to the most natural class of subgroups, namely the relatively quasiconvex subgroups. In this section we introduce these subgroups from several different points of view, corresponding to the various characterizations of relative hyperbolicity. In each setting, we describe a geometrically natural family of "relatively quasiconvex" subgroups. As before, our main goal is to show that the various definitions of relative quasiconvexity are equivalent. As part of this equivalence, we will show that each of the conditions below is an intrinsic property of the subgroup $H$ in the relatively hyperbolic group $(G, \mathbb{P})$, and does not depend on any specific choices made throughout the definition.

Throughout this section, we assume that $G$ is countable, $\mathbb{P}=\left\{P_{1}, \ldots, P_{n}\right\}$ is a finite collection of subgroups, and that $(G, \mathbb{P})$ is relatively hyperbolic.

Definition 6.1 (Quasiconvex subspace) Let $X$ be a geodesic metric space. A subspace $Y \subseteq X$ is $\kappa$-quasiconvex for some $\kappa>0$ if every geodesic of $X$ connecting two points of $Y$ lies in the $\kappa$-neighborhood of $Y$.

Definition 6.2 (QC-1) A subgroup $H \leq G$ is relatively quasiconvex if the following holds. Let $M$ be some (any) compact, metrizable space on which $(G, \mathbb{P})$ acts as a geometrically finite convergence group. Then the induced convergence action of $H$ on the limit set $\Lambda H \subseteq M$ is geometrically finite. 
The next two definitions of relative quasiconvexity allow us to recognize relatively quasiconvex subgroups in the setting of Gromov's original definition of relative hyperbolicity.

To express the first, we need the notion of the join of a set in the boundary.

Definition 6.3 (Join) Let $X$ be $\delta$-hyperbolic, and suppose $\Lambda \subseteq \partial X$ is a subset with at least two points. The join of $\Lambda$, denoted join $(\Lambda)$, is the union of all geodesic lines in $X$ joining pairs of points in $\Lambda$.

Remark 6.4 One should think of the join as a "quasiconvex hull." In fact, it is easy to see that if $X$ is $\delta$-hyperbolic, then join $(\Lambda)$ is a $\kappa$-quasiconvex subspace for some $\kappa=\kappa(\delta)$ (see for instance Gromov [19, Section 7.5.A]). By an elementary argument, join $(\Lambda)$ is quasi-isometric to a geodesic hyperbolic space $Y$ whose boundary $\partial Y$ is canonically homeomorphic to $\Lambda$. Any horofunction on $X$ based at a point of $\Lambda$ restricts to a horofunction on $Y$. Consequently a horoball of $X$ centered in $\Lambda$ restricts to a horoball of $Y$.

Definition 6.5 (QC-2) A subgroup $H \leq G$ is relatively quasiconvex if the following holds. Let $X$ be some (any) proper $\delta$-hyperbolic space on which $(G, \mathbb{P})$ has a cusp uniform action. Then either $H$ is finite, $H$ is parabolic, or $H$ has a cusp uniform action on a geodesic hyperbolic space $Y$ quasi-isometric to the subspace join $(\Lambda H) \subseteq X$.

Definition 6.6 (QC-3) A subgroup $H \leq G$ is relatively quasiconvex if the following holds. Let $(X, \rho)$ be some (any) proper $\delta$-hyperbolic space on which $(G, \mathbb{P})$ has a cusp uniform action. Let $X-U$ be some (any) truncated space for $G$ acting on $X$. For some (any) basepoint $x \in X-U$ there is a constant $\mu \geq 0$ such that whenever $c$ is a geodesic in $X$ with endpoints in the orbit $H x$, we have

$$
c \cap(X-U) \subseteq \mathcal{N}_{\mu}(H x),
$$

where the neighborhood is taken with respect to the metric $\rho$ on $X$.

The following construction was introduced by Farb [15] in the context of manifolds with pinched negative curvature, and further elaborated by Bowditch [7]. Farb credits the "electric" terminology to Thurston.

Definition 6.7 (Electric space) Suppose $(G, \mathbb{P})$ has a cusp uniform action on a $\delta$ hyperbolic space $(X, \rho)$, and let $X-U$ be a truncated space for the action. The electric pseudometric $\hat{\rho}$ on $X$ is the path pseudometric obtained by modifying $\rho$ so that it is identically zero on each horoball of $U$ (essentially collapsing each horoball 
of $U$ to a point). The electric space associated to the pair $(X, U)$ is the pseudometric space $(X, \hat{\rho})$.

A relative path $(\gamma, \alpha)$ in $(X, U)$ is a path $\gamma$ together with a subset $\alpha$ consisting of a disjoint union of finitely many paths $\alpha_{1}, \ldots, \alpha_{n}$ occurring in this order along $\gamma$ such that each $\alpha_{i}$ lies in some closed horoball $B_{i}$ of $U$. We also assume that $B_{i} \neq$ $B_{i+1}$. The relative length of $(\gamma, \alpha)$ is the length in $X$ of $\gamma-\alpha$. We write $\gamma=$ $\beta_{0} \cup \alpha_{1} \cup \beta_{1} \cup \cdots \cup \alpha_{n} \cup \beta_{n}$, where the $\beta_{i}$ are the complementary paths of $\gamma$. (Either of $\beta_{0}$ or $\beta_{n}$ could be a trivial path.) A relative path $(\gamma, \alpha)$ is efficient if $B_{i} \neq B_{j}$ for $i \neq j$, and is semipolygonal if each $\alpha_{i}$ is a geodesic of $X$.

There are natural notions of a relative path $(\gamma, \alpha)$ being an electric geodesic or an electric $\epsilon$-quasigeodesic given by comparing the relative length of an arbitrary subsegment with the electric distance between its endpoints in the obvious way.

The following result, roughly speaking, says that an electric quasigeodesic is also a quasigeodesic in $(X, d)$.

Lemma 6.8 [7, Lemma 7.3] Given constants $\delta, \epsilon>0$, there are constants $r=r(\delta)$ and $\epsilon^{\prime}=\epsilon^{\prime}(\delta, \epsilon)$ so that the following holds. Suppose $(G, \mathbb{P})$ has a cusp uniform action on a $\delta$-hyperbolic space $(X, \rho)$. Let $X-U$ be a truncated space for the action such that any two horoballs of $U$ are separated in $X$ by a $\rho$-distance at least $r$, and let $\hat{\rho}$ be the corresponding electric metric. If $(\gamma, \alpha)$ is an efficient, semipolygonal relative path in $(X, U)$ and also an electric $\epsilon$-quasigeodesic then $\gamma$ is an $\epsilon^{\prime}$-quasigeodesic in $X$.

Roughly speaking the following definition says that a subgroup is relatively quasiconvex if its orbits are quasiconvex with respect to electric geodesics.

Definition 6.9 (QC-4) A subgroup $H \leq G$ is relatively quasiconvex provided that the following holds. Let $(G, \mathbb{P})$ have a cusp uniform action on some (any) $\delta$-hyperbolic space $(X, d)$, and choose some (any) truncated space $X-U$ for the action. Suppose each pair of horoballs of $U$ is separated by at least a distance $r$, where $r=r(\delta)$ is the constant given by Lemma 6.8 .

For some (each) basepoint $x \in X-U$ there is a constant $\kappa>0$ so that for every efficient, semipolygonal relative path $(\gamma, \alpha)$ that is also an electric geodesic connecting points of $H x$, the subset $\gamma-\alpha$ lies in the $\kappa$-neighborhood of $H x$ in $(X, \rho)$.

Observe that a similar condition will hold for electric quasigeodesics connecting points of the orbit $H x$, since Lemma 6.8 implies that electric quasigeodesics with common endpoints track Hausdorff close in $X$. 
The next definition is quite close to the one introduced by Osin for subgroups of a finitely generated relatively hyperbolic group. The only difference between Osin's definition and the one below is that we use a proper, left invariant metric $d$ on $G$ where Osin used the word metric for a finite generating set. It is not clear whether one can use the word metric for a finite relative generating set in place of the proper, left invariant metric.

Definition 6.10 (QC-5) A subgroup $H \leq G$ is relatively quasiconvex if the following holds. Let $\mathcal{S}$ be some (any) finite relative generating set for $(G, \mathbb{P})$, and let $\mathcal{P}$ be the union of all $P_{i} \in \mathbb{P}$. Consider the Cayley graph $\bar{\Gamma}=\operatorname{Cayley}(G, \mathcal{S} \cup \mathcal{P})$ with all edges of length one. Let $d$ be some (any) proper, left invariant metric on $G$. Then there is a constant $\kappa=\kappa(\mathcal{S}, d)$ such that for each geodesic $\bar{c}$ in $\bar{\Gamma}$ connecting two points of $H$, every vertex of $\bar{c}$ lies within a $d$-distance $\kappa$ of $H$.

We will see that the preceding definition is independent of the choice of finite relative generating set $\mathcal{S}$ and the choice of proper metric $d$. In particular, if $\mathcal{S}$ is a finite generating set for $G$ in the traditional sense, then we can choose $d$ to be the word metric for $\mathcal{S}$. Thus in the finitely generated case, $(\mathrm{QC}-5)$ is equivalent to the definition given by Osin in [27].

\section{Relative quasiconvexity: Equivalence of definitions}

We now establish the equivalence of the various definitions of relative quasiconvexity introduced in the previous section. Throughout this section $(G, \mathbb{P})$ is a relatively hyperbolic group and $H$ is any subgroup of $G$.

Proposition 7.1 Definitions (QC-1) and (QC-2) are well-defined and are equivalent.

The proof uses the following result due to Bowditch.

Theorem 7.2 [7, Theorem 9.4] Suppose $(G, \mathbb{P})$ has cusp uniform actions on spaces $X$ and $X^{\prime}$. Then $\partial X$ and $\partial X^{\prime}$ are $G$-equivariantly homeomorphic.

Proof of Proposition 7.1 By Corollary 5.3, if $(G, \mathbb{P})$ has a geometrically finite convergence action on a compact, metrizable space $M$, then $(G, \mathbb{P})$ acts geometrically finitely on a proper $\delta$-hyperbolic space $X$ and $M$ is $G$-equivariantly homeomorphic to the boundary of $X$. It follows from Theorem 7.2 that condition $(\mathrm{QC}-1)$ does not depend on the choice of compactum $M$ or the choice of geometrically finite action of $(G, \mathbb{P})$ on $M$. 
Suppose $(G, \mathbb{P})$ has a cusp uniform action on a proper $\delta$-hyperbolic space $X$. Then the induced action on $M=\partial X$ is a geometrically finite convergence action. We will show that (QC-1) holds for the action of $G$ on $M$ if and only if (QC-2) holds for the action of $G$ on $X$. By the preceding paragraph, it follows immediately that (QC-2) is independent of the choice of $X$.

Recall our convention that the unique action of a finite group $H$ on the empty set is a convergence group action. Therefore each finite subgroup $H \leq G$ satisfies both (QC-1) and (QC-2). Similarly, the unique action of a countable group $H$ on a single point is a convergence group action. If $H$ is parabolic then $\Lambda H$ is a single point, and hence $H$ satisfies both (QC-1) and (QC-2).

Now suppose $H$ is neither finite nor parabolic. Then $\Lambda H \subseteq \partial X$ contains at least two points, and join $(\Lambda H)$ is a nonempty quasiconvex subspace of $X$ invariant under the action of $H$. By Remark 6.4, the subspace join $(\Lambda H)$ with the subspace metric is quasi-isometric to a $\delta^{\prime}$-hyperbolic geodesic space $Y$ for some $\delta^{\prime} \geq 0$, and $\partial Y$ is $H$-equivariantly homeomorphic to $\Lambda H \subseteq \partial X$.

Applying Theorem 5.4 to $Y$, we see that (QC-1) holds for $\partial Y=\Lambda H$ if and only if (QC-2) holds for $Y$.

Lemma 7.3 Let $(G, \mathbb{P})$ have a cusp uniform action on a $\delta$-hyperbolic space $(X, \rho)$ with associated truncated space $X-U$. For each $N$ there is a constant $M_{1}$ so that the following holds. Choose two $\rho$-geodesics $c, c^{\prime}$ such that

$$
\max \left\{\rho\left(c_{-}, c_{-}^{\prime}\right), \rho\left(c_{+}, c_{+}^{\prime}\right)\right\}<N .
$$

If $c \cap(X-U)$ and $c^{\prime} \cap(X-U)$ are both nonempty then the Hausdorff $\rho$-distance between $c \cap(X-U)$ and $c^{\prime} \cap(X-U)$ is less than $M_{1}$.

Proof The geodesics $c$ and $c^{\prime}$ are opposite sides of a $2 \delta$-thin geodesic quadrilateral. It follows that the Hausdorff distance in $X$ between $c$ and $c^{\prime}$ is at most $N^{\prime}:=N+2 \delta$. Choose a point $p \in c \cap(X-U)$. Then $p$ is within a distance $N^{\prime}$ of a point $q \in c^{\prime}$. Observe that $q$ also lies in $\mathcal{N}_{N^{\prime}}(X-U)$. By Lemma 2.1 there is a segment of $c^{\prime}$ from $q$ to $X-U$ of length at most $M_{0}$, where $M_{0}$ depends only on $X-U$ and $N^{\prime}$. Therefore $p$ lies within a distance $N^{\prime}+M$ of a point $r \in c^{\prime} \cap(X-U)$. Interchanging the roles of $c$ and $c^{\prime}$ completes the proof.

Lemma 7.4 Let $(G, \mathbb{P})$ have a cusp uniform action on a $\delta$-hyperbolic space $(X, \rho)$. Let $X-U$ and $X-U^{\prime}$ be two corresponding truncated spaces. Then there is a constant $M_{2}$ such that for each $\rho$-geodesic $c$ in $X$, if $c \cap(X-U)$ and $c \cap\left(X-U^{\prime}\right)$ are both nonempty then the Hausdorff $\rho$-distance between $c \cap(X-U)$ and $c \cap\left(X-U^{\prime}\right)$ is at most $M_{2}$. 
Proof Recall that the horoballs of $U$ and $U^{\prime}$ lie in finitely many $G$-orbits, corresponding to the finitely many peripheral subgroups of $\mathbb{P}$. By shrinking the horoballs of $U$ and $U^{\prime}$ equivariantly, we can obtain a third truncated space $X-V$ such that $V \subseteq U \cap U^{\prime}$. If $c$ has nonempty intersection with each of the given truncated spaces then it also has nonempty intersection with $X-V$. Thus it suffices to prove the lemma in the special case when $U^{\prime} \subseteq U$. Moreover, it is enough to consider the further special case that $U$ and $U^{\prime}$ differ on only one orbit of horoballs, since this case can be iterated finitely many times to obtain the desired result.

Suppose $U$ and $U^{\prime}$ agree on all but one orbit, and this exceptional orbit is represented by horoballs $B$ and $B^{\prime}$ of $U$ and $U^{\prime}$ respectively, such that $B^{\prime} \subset B$. Then $X-U \subset X-U^{\prime}$. Clearly we have

$$
c \cap(X-U) \subseteq c \cap\left(X-U^{\prime}\right) .
$$

On the other hand, any subsegment $\bar{c}$ of $c$ that lies in $X-U^{\prime}$ but not in $X-U$ is a translate of a geodesic in $B-B^{\prime}$. By Lemma 2.2, such a segment has length at most $M_{1}=M_{1}\left(B, B^{\prime}\right)$. Thus

$$
c \cap\left(X-U^{\prime}\right) \subseteq \mathcal{N}_{M_{0}}(c \cap(X-U)),
$$

completing the proof.

Proposition 7.5 Suppose $(G, \mathbb{P})$ has a cusp uniform action on $(X, \rho)$, and $H \leq G$. If $H$ satisfies (QC-3) with respect to one choice of truncated space $X-U$ and basepoint $x \in X-U$, then $H$ satisfies (QC-3) with respect to any other truncated space $X-U^{\prime}$ and basepoint $x^{\prime}$.

Proof Let us first consider the effect of changing the basepoint $x \in X-U$ to another point $x^{\prime} \in X-U$ in the same truncated space. Choose $h_{0}, h_{1} \in H$, a $\rho$-geodesic $c$ from $h_{0}(x)$ to $h_{1}(x)$, and a $\rho$-geodesic $c^{\prime}$ from $h_{0}\left(x^{\prime}\right)$ to $h_{1}\left(x^{\prime}\right)$. Then

$$
\rho\left(c_{-}, c_{-}^{\prime}\right)=\rho\left(c_{+}, c_{+}^{\prime}\right)=\rho\left(x, x^{\prime}\right) .
$$

By Lemma 7.3, the Hausdorff distance between $c \cap(X-U)$ and $c^{\prime} \cap(X-U)$ is at most $M_{1}$ for some constant $M_{1}$ that depends on $x$ and $x^{\prime}$ but not on the choice of $h_{0}$ and $h_{1}$. If $c \cap(X-U)$ lies in $\mathcal{N}_{\kappa}(H x)$ then $c^{\prime} \cap(X-U)$ lies in $\mathcal{N}_{\kappa+M_{1}}\left(H x^{\prime}\right)$. Therefore if we fix $X-U$, then (QC-3) does not depend on the choice of basepoint $x \in X-U$.

Now suppose $X-U$ and $X-U^{\prime}$ are two truncated spaces corresponding to the action of $G$ on $X$. Choose basepoints $x \in X-U$ and $x^{\prime} \in X-U^{\prime}$. Shrinking horoballs, we can obtain a third truncated space $X-V$ with $V \subseteq U \cap U^{\prime}$. In particular, note that $x$ and $x^{\prime}$ both lie in $X-V$. If $H$ satisfies (QC-3) with respect to $X-U$ with 
basepoint $x$, then by Lemma 7.4 it also satisfies (QC-3) with respect to $X-V$ with basepoint $x$. By the preceding paragraph, $H$ also satisfies (QC-3) with respect to $X-V$ and basepoint $x^{\prime}$. Finally, another application of Lemma 7.4 shows that $H$ satisfies (QC-3) with respect to $X-U^{\prime}$ and basepoint $x^{\prime}$.

Thus (QC-3) is independent of the choice of truncated space $X-U$ and independent of the choice of basepoint $x \in X-U$.

Proposition 7.6 Definition (QC-3) is well-defined and is equivalent to Definition (QC-2).

Proof In Proposition 7.5 we showed that for each fixed cusp uniform action of $(G, \mathbb{P})$ on a space $(X, \rho)$, whether a subgroup $H$ satisfies $(\mathrm{QC}-3)$ does not depend on the choice of truncated space and basepoint. In Proposition 7.1 we showed that (QC-2) does not depend on the choice of space $X$ or the choice of cusp uniform action of $(G, \mathbb{P})$ on $X$. Thus, in order to show that $(\mathrm{QC}-3)$ is also independent of the space $X$ and cusp uniform action, it suffices to show that (QC-2) holds for a particular action of $G$ on $X$ if and only if (QC-3) holds for the same action with respect to some choice of truncated space $X-U$ and basepoint $x \in X-U$.

$(\mathrm{QC}-2) \Rightarrow(\mathrm{QC}-3) \quad$ Choose any truncated space $X-U$ for the action of $G$ on $X$. If $H$ is finite, then (QC-3) is immediate since the orbit $H x$ is bounded. If $H$ is parabolic with parabolic fixed point $p \in \partial X$, and $x \in X-U$ is any basepoint, then there is a horofunction based at $p$ that is identically zero on the orbit $H x$. Let $B$ be the horoball of $U$ centered at $p$. By Lemma 2.3, there is a constant $M_{2}$ such that for each geodesic $c=[a, b]$ connecting two points of $H x$, we have

$$
c \cap(X-U) \subseteq \mathcal{N}_{M_{2}}(\{a, b\}) .
$$

Thus (QC-3) holds for $H$.

Now suppose $\Lambda H$ contains at least two points. Then join $(\Lambda H)$ is nonempty and $\kappa$-quasiconvex for some $\kappa>0$. Suppose the action of $H$ on $Y$ is cusp uniform, where $Y$ is quasi-isometric to join $(\Lambda H)$. Under this quasi-isometry, every horoball of $Y$ pulls back to a subset of join $(\Lambda H)$ of the form $B \cap$ join $(\Lambda H)$ for some horoball $B$ of $X$. Therefore, the action of $H$ on join $(\Lambda H)$ is "cusp uniform" in the sense that $X$ contains a union of horoballs $U^{\prime}$ centered at the parabolic points of $H$ such that the horoballs lie in finitely many $H$-orbits and such that $H$ acts cocompactly on the "truncated space" join $(\Lambda H)-U^{\prime}$.

Shrink the horoballs of $U^{\prime} H$-equivariantly so that $\rho\left(U^{\prime}, X-U\right)>\kappa$. Then $H$ still acts cocompactly on join $(\Lambda H)-U^{\prime}$. Let $\nu<\infty$ be the $\rho$-diameter of a compact set $K \subseteq X$ 
whose $H$-translates cover join $(\Lambda H)-U^{\prime}$. Choose a basepoint $x \in$ join $(\Lambda H)-U^{\prime}$, and let $c$ be a geodesic in $X$ connecting two points of $H x$. Since the endpoints of $c$ lie in join $(\Lambda H)$, the $\kappa$-quasiconvexity of join $(\Lambda H)$ implies that each $p \in c \cap(X-U)$ is within a distance $\kappa$ of a point $q \in$ join $(\Lambda H)$. Observe that $q$ is also in $\mathcal{N}_{\kappa}(X-U)$. By our choice of $U^{\prime}$, it follows that both $x$ and $q$ lie in join $(\Lambda H)-U^{\prime}$. Therefore, for some $h \in H$, we have $\rho(h x, q)<v$, establishing (QC-3).

$(\mathrm{QC}-3) \Rightarrow(\mathrm{QC}-2)$ Suppose $(G, \mathbb{P})$ has a cusp uniform action on $(X, \rho)$, and $H \leq G$ is infinite and not parabolic. Then join $(\Lambda H)$ is nonempty and $\kappa$-quasiconvex for some $\kappa>0$, and quasi-isometric to a hyperbolic geodesic space $Y$. In order to establish (QC-2), it suffices to show that the action of $H$ on $Y^{\prime}:=\operatorname{join}(\Lambda H)$ is cusp uniform in the above sense.

Choose a truncated space $X-U$ for the cusp uniform action of $G$ on $X$ and a basepoint $x \in Y^{\prime}-U$ such that $H$ satisfies (QC-3) with respect to $X-U$ and $x$ using quasiconvexity constant $\mu$.

Then any geodesic $c$ connecting two points of $H x$ satisfies

$$
c \cap(X-U) \subseteq \mathcal{N}_{\mu}(H x)
$$

If $\bar{c}$ is a bi-infinite geodesic in $X$ obtained as a pointwise limit of such segments $c$, then $\bar{c}$ connects two points of $\Lambda H$ and satisfies

$$
\bar{c} \cap(X-U) \subseteq \mathcal{N}_{\mu}(H x) .
$$

Since $X$ is $\delta$-hyperbolic, and each point of $\Lambda H$ is a limit point of $H x$, every geodesic connecting two points of $\Lambda H$ lies in the $2 \delta$-neighborhood of such a geodesic $\bar{c}$. (This is because any two bi-infinite geodesics with the same endpoints at infinity in a $\delta$-hyperbolic space have Hausdorff distance at most $2 \delta$.) Therefore

$$
Y^{\prime}-U \subseteq \mathcal{N}_{2 \delta+\mu}(H x),
$$

proving that $H$ acts cocompactly on $Y^{\prime}-U$.

In order to establish (QC-2), we must show that $H$ acts cocompactly on $Y^{\prime}-U^{\prime}$, where $U^{\prime}$ is an $H$-equivariant family of horoballs centered only at the parabolic points of $H$. It suffices to show that the horoballs $B$ of $U$ that meet $Y^{\prime}$ and are not based in $\Lambda H$ have uniformly bounded intersections with $Y^{\prime}$. Since then $Y^{\prime}-U$ is quasidense in $Y^{\prime}-U^{\prime}$.

If a horoball $B$ of $U-U^{\prime}$ meets $Y^{\prime}$, then it also meets $Y^{\prime}-U$, which lies in $\mathcal{N}_{2 \delta+\mu}(H x)$. Hence, $\rho(B, H x)$ is at most $2 \delta+\mu$. Then the translate $h B$ of $B$ by some element $h \in H$ intersects $B(x, 2 \delta+\mu)$. Since only finitely many horoballs of $U$ 
meet any metric ball in $X$, the horoballs of $U$ meeting $Y^{\prime}$ must lie in only finitely many $H$-orbits. If such a horoball is not based in $\Lambda H$, then its intersection with $Y^{\prime}$ is bounded. Combining these bounds for the finitely many $H$-orbits gives a uniform bound on the diameter of all such intersections, which completes the proof.

Proposition 7.7 Definition (QC-4) is well-defined and equivalent to (QC-3).

Proof Recall that we have shown that if a subgroup $H \leq G$ satisfies (QC-3) with respect to a cusp uniform action, truncated space, and basepoint, then it satisfies (QC-3) with respect to any other such choices. Choose a proper $\delta$-hyperbolic space $(X, \rho)$, a cusp uniform action of $(G, \mathbb{P})$ on $X$, a truncated space $X-U$, and a basepoint $x \in X-U$. Suppose further that the horoballs of $U$ are pairwise separated by a $\rho$-distance at least $r$, where $r=r(\delta)$ is the constant provided by Lemma 6.8. We will show that $H$ satisfies (QC-3) with respect to these choices if and only if it satisfies (QC-4).

More specifically, we will show that there is a constant $L$ such that the following holds. Let $c$ be a $\rho$-geodesic and $(\gamma, \alpha)$ a semipolygonal relative path that is also an electric geodesic. If $c$ and $\gamma$ have the same endpoints in $H x$, then the Hausdorff $\rho$-distance between $c \cap(X-U)$ and $\gamma-\alpha$ is at most $L$.

By Lemma 6.8, the path $\gamma$ is an $\epsilon$-quasigeodesic in $(X, \rho)$ for some uniform constant $\epsilon$. By the Morse Lemma, the Hausdorff $\rho$-distance between $c$ and $\gamma$ is at most $\eta=\eta(\delta, \epsilon)$. Since $(\gamma, \alpha)$ is an electric geodesic with respect to $(X, U)$, the subset $\gamma-\alpha$ must lie in $X-U$. Therefore any point $p \in \gamma-\alpha$ is within a $\rho$-distance $\eta$ of a point $q \in c \cap \mathcal{N}_{\eta}(X-U)$. By Lemma 2.1 the point $q$ is within a $\rho$-distance $M_{0}$ of a point $q^{\prime} \in c \cap(X-U)$, where $M_{0}$ depends only on $\eta$ and $U$. (Here we are using that $\mathcal{N}_{\eta}(X-U)$ differs from $X-U$ on only finitely many orbits of horoballs.) A similar argument (using a quasigeodesic variation of Lemma 2.1) bounds the distance from an arbitrary point of $\gamma-\alpha$ to $c \cap(X-U)$. Thus we have an upper bound on the Hausdorff $\rho$-distance between $\gamma-\alpha$ and $c \cap(X-U)$. It is now clear that if either set lies near $H x$, then so does the other.

It is a well-known result, first observed by Efromovich [14] that if $X$ is a connected length space, and $G$ is a group acting metrically properly, coboundedly and isometrically on $X$, then $G$ is finitely generated and quasi-isometric to $X$. Recall that a length space is connected if and only if all distances are finite. The following result dealing with actions on arbitrary length spaces is an easy corollary to Efromovich's Theorem.

Proposition 7.8 (Disconnected Efromovich's Theorem) Let $X$ be a length space (possibly disconnected). Let $G$ be a countable group with proper, left invariant metric $d$ 
acting metrically properly, coboundedly and isometrically on $X$. Let $Y$ be a component of $X$. Then the $G$-translates of $Y$ cover $X$, the stabilizer $H$ of $Y$ has a finite generating set $\mathcal{S}$, and for each basepoint $x_{0} \in X$, the map $g \mapsto g\left(x_{0}\right)$ induces a quasi-isometry from the (possibly disconnected) Cayley graph Cayley $(G, \mathcal{S})$ to $X$.

Proof Since any two components of $X$ are separated by an infinite distance, it is clear that $H$ acts coboundedly on $Y$. Applying Efromovich's theorem to the action of $H$ on the connected length space $Y$ gives a finite generating set $\mathcal{S}$ for $H$ and shows that Cayley $(H, \mathcal{S})$ is quasi-isometric to $Y$. Note that if $H \neq G$, then Cayley $(G, \mathcal{S})$ and $X$ are both disconnected with all components separated by infinite distances. Since $G$ acts on $X$ with a connected quotient, the translates of $Y$ cover $X$. In other words, the path components of $X$ are precisely the translates of $Y$, which are in one-to-one correspondence with the left cosets $g H$ of $H$ in $G$, which in turn correspond to the path components in the Cayley graph.

Proposition 7.9 Suppose $(G, \mathbb{P})$ is relatively hyperbolic with finite relative generating set $\mathcal{S}$. Let $(G, \mathbb{P})$ have a cusp uniform action on $(X, \rho)$ with associated truncated space $X-U$. Suppose the horoballs of $U$ are pairwise separated by some minimum positive distance $r$.

For each basepoint $x \in X-U$ the orbit map $g \mapsto g(x)$ extends to a quasi-isometry

$$
f: \operatorname{Cayley}(G, \mathcal{S} \cup \mathcal{P}) \rightarrow(X, \hat{\rho}),
$$

where $\hat{\rho}$ denotes the electric pseudometric for $(X, U)$. Furthermore, $f$ can be chosen so that each geodesic edge-path in the Cayley graph maps to an efficient semipolygonal relative path in $(X, U)$.

Proof Choose a basepoint $x \in X-U$, and let $Z$ be the rectifiable-path component of $X-U$ containing $x$. Then $Z$ is connected when endowed with the induced length metric. Let $Y$ be the union of all $G$-translates of $Z$. Since $G$ acts cocompactly on $(X-U, \rho)$ and $Y$ is a nonempty, $G$-equivariant subspace of $X-U$, it follows that $Y$ is quasidense in $(X-U, \rho)$. Therefore, $G$ acts on $Y$ with a rectifiable-path connected quotient. Let $\bar{\rho}$ denote the length metric on $Y$ induced by $\rho$. Then $G$ acts coboundedly, metrically properly and isometrically on $(Y, \bar{\rho})$. By Proposition 7.8, the stabilizer $H$ of $Z$ is generated by a finite set $\mathcal{T}$, and the orbit map $g \mapsto g(x)$ induces a quasi-isometry Cayley $(G, \mathcal{T}) \rightarrow(Y, \bar{\rho})$. Under this quasi-isometry, the set of left cosets $g P$ for all $g \in G$ and all $P \in \mathbb{P}$ corresponds with the set of horoballs in $U$. The electric space $(X, \hat{\rho})$ is quasi-isometric to its quasidense subspace $(Y, \hat{\rho})$, which in turn is clearly quasi-isometric to $\operatorname{Cayley}(G, \mathcal{T} \cup \mathcal{P})$. In particular, since the electric space is connected, this Cayley graph is also connected, establishing that $\mathcal{T}$ is a relative 
generating set for $(G, \mathbb{P})$. By an elementary argument (see, for instance, Osin [27, Proposition 2.8]), the identity $G \rightarrow G$ induces a quasi-isometry

$$
\operatorname{Cayley}(G, \mathcal{S} \cup \mathcal{P}) \rightarrow \operatorname{Cayley}(G, \mathcal{T} \cup \mathcal{P})
$$

for any other relative generating set $\mathcal{S}$. Composing these quasi-isometries gives a quasi-isometry

$$
f: \operatorname{Cayley}(G, \mathcal{S} \cup \mathcal{P}) \rightarrow(X, \hat{\rho})
$$

mapping $g \mapsto g(x)$ for each $g \in G$.

Since $G$ is quasidense in the Cayley graph, we can modify $f$ so that it sends the edges of the Cayley graph to any relative paths we like, provided that the relative lengths of these paths are uniformly bounded. For each generator $s \in \mathcal{S}$, choose a $\rho$-geodesic $\beta_{s}$ from $x$ to $s(x)$, considered as a relative path $(\gamma, \alpha)$ with $\alpha$ empty. Let $f$ map each Cayley graph edge labeled by $s$ to the appropriate translate of $\beta$. For each $P \in \mathcal{P}$, choose a $\rho$-geodesic $\beta_{P}$ from $x$ to the horoball $B$ stabilized by $P$. For each generator labeled by $p \in P$, let $\left(\gamma_{p}, \alpha_{p}\right)$ be the relative path with $\gamma_{p}:=\beta_{P} \cup \alpha_{p} \cup \overline{\left(p \circ \beta_{P}\right)}$, where $\alpha_{p}$ is a $\rho$-geodesic path in $B$ from the terminal point $b$ of $\beta_{P}$ to the point $p(b)$, and where the bar indicates following a path in the reverse direction. Let $f$ map each Cayley graph labeled by $p$ to the appropriate translate of $\left(\gamma_{p}, \alpha_{p}\right)$.

Observe that each edge path in the Cayley graph is mapped by $f$ to a semipolygonal relative path. Furthermore, if the image $(\gamma, \alpha)$ is not efficient, and $\alpha$ is a disjoint union of paths $\alpha_{1}, \ldots, \alpha_{n}$, then for some $i<j$ the paths $\alpha_{i}$ and $\alpha_{j}$ lie in the same horoball $B$. Let $e_{i}$ and $e_{j}$ be the corresponding edges in the Cayley graph. It follows that the initial point $v$ of $e_{i}$ and the terminal point $w$ of $e_{j}$ lie in the same left coset $g P$ of some $P \in \mathbb{P}$. In particular, in the Cayley graph, the distance from $v$ to $w$ is at most 1 , so the given edge path cannot be geodesic.

Proposition 7.10 Definition (QC-5) is well-defined and equivalent to (QC-4).

Proof Fix a finite relative generating set $\mathcal{S}$ for $(G, \mathbb{P})$, and suppose $H$ satisfies $(\mathrm{QC}-5)$ with respect to $\mathcal{S}$ and some proper left-invariant metric $d$ with quasiconvexity constant $\kappa=\kappa(\mathcal{S}, d)$. We first show that (QC-5) continues to hold if we replace $d$ with another proper left-invariant metric $d^{\prime}$. Let $\mathcal{B}$ be the finite ball of radius $\kappa$ centered at the identity in the metric space $(G, d)$. Let

$$
\kappa^{\prime}:=\max \left\{d^{\prime}(1, g) \mid g \in \mathcal{B}\right\}+1<\infty .
$$

We will see that $(\mathrm{QC}-5)$ holds for $\mathcal{S}$ and $d^{\prime}$ with $\kappa\left(\mathcal{S}, d^{\prime}\right):=\kappa^{\prime}$. Let $\bar{c}$ be any geodesic in Cayley $(G, \mathcal{S} \cup \mathcal{P})$ connecting two points of $H$, and let $v \in G$ be a vertex of $c$. Then there is an element $w \in H$ such that $d(v, w)<\kappa$. Since $d$ is left-invariant, 
$d\left(1, v^{-1} w\right)<\kappa$ so that $v^{-1} w \in \mathcal{B}$. But then $d^{\prime}(v, w)=d^{\prime}\left(1, v^{-1} w\right)<\kappa^{\prime}$ as desired, establishing (QC-5) for $\mathcal{S}$ and $d^{\prime}$. Note that the above argument holds even if $d$ or $d^{\prime}$ is a pseudometric (rather than a metric) provided that both are proper and left-invariant.

Now let us consider the equivalence of $(\mathrm{QC}-4)$ and $(\mathrm{QC}-5)$. Fix a finite relative generating set $\mathcal{S}$ for $(G, \mathbb{P})$ and a cusp uniform action of $(G, \mathbb{P})$ on a proper $\delta$ hyperbolic space $(X, \rho)$ with an associated truncated space $X-U$. Suppose further that the horoballs of $U$ are pairwise separated by at least a distance $r$, where $r=r(\delta)$ is the constant given by Lemma 6.8. Fix a basepoint $x \in X-U$. Define a proper left-invariant pseudometric $d_{G}$ on $G$ using distances in $X$ between orbit points; that is, we set

$$
d_{G}\left(g_{1}, g_{2}\right):=\rho\left(g_{1}(x), g_{2}(x)\right) \text {. }
$$

To prove the proposition, it suffices to show that (QC-5) holds for $\mathcal{S}$ and $d_{G}$ if and only if (QC-4) holds for $X, U$, and $x$. Since $\mathcal{S}$ is arbitrary, it therefore follows that $(\mathrm{QC}-5)$ is independent of the choice of $\mathcal{S}$.

$(\mathrm{QC}-4) \Rightarrow(\mathrm{QC}-5) \quad$ By Proposition 7.9, there is an $\epsilon$-quasi-isometry

$$
f: \operatorname{Cayley}(G, \mathcal{S} \cup \mathcal{P}) \rightarrow(X, \hat{\rho})
$$

for some $\epsilon>0$, such that $f$ maps $g \in G$ to $g(x)$ and such that each geodesic $c$ in Cayley $(G, \mathcal{S} \cup \mathcal{P})$ with endpoints in $H$ maps to an efficient, semipolygonal relative $\epsilon$-quasigeodesic $(\gamma, \alpha)$ in $\left(X, U^{\prime}\right)$ such that $\gamma$ has endpoints in $H x$. Let $\left(\gamma^{\prime}, \alpha^{\prime}\right)$ be an efficient, semipolygonal relative geodesic such that $\gamma^{\prime}$ has the same endpoints as $\gamma$. By Lemma 6.8, $\gamma$ and $\gamma^{\prime}$ are $\epsilon^{\prime}$-quasigeodesics in $(X, \rho)$ for some $\epsilon^{\prime}$ depending on $\epsilon$. In particular, by the Morse Lemma the Hausdorff $\rho$-distance between $\gamma$ and $\gamma^{\prime}$ is at most $L=L\left(\delta, \epsilon^{\prime}\right)$. As in the proof of Proposition 7.7 there is a uniform upper bound $L^{\prime}$ on the Hausdorff $\rho$-distance between $\gamma-\alpha$ and $\gamma^{\prime}-\alpha^{\prime}$.

Choose an arbitrary vertex $g$ of $c$. Being a vertex of the Cayley graph, $g$ is an element of $G$. Thus $f$ maps $g$ to the orbit point $g(x) \in \gamma-\alpha$, which is within a $\rho$-distance $L^{\prime}$ of some $y \in \gamma^{\prime}-\alpha^{\prime}$. By (QC-4), the point $y$ is within a $\rho$-distance $\kappa$ of $H x$. Applying the triangle inequality shows that $\rho(g(x), H x)<L+\kappa$. By our definition of $d_{G}$, we also have $d_{G}(g, H)<L+\kappa$, establishing (QC-5).

$(\mathrm{QC}-5) \Rightarrow(\mathrm{QC}-4)$ Let $\left(\gamma^{\prime}, \alpha^{\prime}\right)$ be an efficient, semipolygonal relative geodesic in $(X, U)$ such that $\gamma^{\prime}$ has endpoints $h x$ and $h^{\prime} x$. Let $c$ be a geodesic in $\operatorname{Cayley}(G, \mathcal{S} \cup \mathcal{P})$ with endpoints $h$ and $h^{\prime}$. As above, $f$ maps $c$ to an efficient, semipolygonal relative $\epsilon$-quasigeodesic $(\gamma, \alpha)$, and the Hausdorff $\rho$-distance between $\gamma-\alpha$ and $\gamma^{\prime}-\alpha^{\prime}$ is bounded above by a constant $L^{\prime}$ that does not depend on the choice of $h, h^{\prime} \in H$. Each point $y \in \gamma^{\prime}-\alpha^{\prime}$ is within a $\rho$-distance $L^{\prime}$ of a point $z \in \gamma-\alpha$, which is within a $\rho$-distance $\epsilon$ of $g x$ for some vertex $g$ of $c$. By (QC-5), we have $d_{G}(g, H)<\kappa$. Thus 
by the definition of $d_{G}$, we have $\rho(g(x), H x)<\kappa$ as well. By the triangle inequality it follows that

$$
\rho(y, H x)<L^{\prime}+\epsilon+\kappa,
$$

establishing (QC-4).

\section{Relative quasiconvexity in the word metric}

In this section we characterize relatively quasiconvex subgroups of finitely generated relatively hyperbolic groups in terms of the geometry of the word metric. We emphasize that throughout this section $(G, \mathbb{P})$ always denotes a finitely generated relatively hyperbolic group, and $\mathcal{S}$ is always a finite generating set for $G$ in the traditional sense. As above, $\mathcal{P}$ denotes the union of the peripheral subgroups $P \in \mathbb{P}$.

If $c$ is a path in Cayley $(G, \mathcal{S} \cup \mathcal{P})$, a lift of $c$ is a path formed from $c$ by replacing each edge of $c$ labelled by an element of $\mathcal{P}$ with a geodesic in Cayley $(G, \mathcal{S})$. The $\mathcal{S}$-edges are left unchanged.

If $c$ is a path in Cayley $(G, \mathcal{S})$ and $M>0$, the $M$-saturation of $c$ is the union of $c$ together with all peripheral cosets $g P$ such that $d_{\mathcal{S}}(c, g P)<M$.

We will need to use several results due to Druțu-Sapir [13] describing the geometry of the word metric for a finitely generated relatively hyperbolic group. These results are collected below.

The first result states that neighborhoods of peripheral subgroups are quasiconvex.

Theorem 8.1 [13, Lemma 4.15] Let $(G, \mathbb{P})$ be a relatively hyperbolic group with finite generating set $\mathcal{S}$. For each $A_{0}$ there is a constant $A_{1}=A_{1}\left(A_{0}\right)$ such that the following holds in Cayley $(G, \mathcal{S})$. Let $c$ be a geodesic segment whose endpoints lie in the $A_{0}$-neighborhood of a peripheral subgroup $P \in \mathbb{P}$. Then $c$ lies in the $A_{1}$-neighborhood of $P$.

In particular, each peripheral subgroup $P \in \mathbb{P}$ is quasiconvex with respect to $\mathcal{S}$. It follows easily that conjugates of peripheral subgroups are quasiconvex as well.

Corollary 8.2 For each $P \in \mathbb{P}$ and $g \in G$, the subgroup $g g^{-1}$ is quasiconvex with respect to $\mathcal{S}$.

Proof Translating the result of Theorem 8.1 by $g$, we see that neighborhoods of $g P$ are quasiconvex. Now the Hausdorff distance between $g P$ and $g P g^{-1}$ is bounded above by $A_{0}:=|g|_{\mathcal{S}}$. Thus any geodesic $c$ with endpoints in $g \mathrm{Pg}^{-1}$ lies in the $A_{1}-$ neighborhood of $g P$, for $A_{1}=A_{1}\left(A_{0}\right)$. Hence $c$ lies in the $\left(A_{1}+A_{0}\right)$-neighborhood of $g P g^{-1}$. 
Quasiconvexity of peripheral subgroups (and their conjugates) has the following consequences using well-known results of Short [30]. We remark that finite generation of peripheral subgroups was first proved by Osin [27] prior to the work of Druțu and Sapir.

Corollary 8.3 (Osin, Druț-Sapir) If $(G, \mathbb{P})$ is relatively hyperbolic with a finite generating set, then for each $P \in \mathbb{P}$ and $g \in G$, the conjugate $g P^{-1}$ has a finite generating set $\mathcal{T}$. Furthermore each conjugate $\mathrm{gPg}^{-1}$ is undistorted in the sense that the inclusion $\mathrm{gPg}^{-1} \hookrightarrow G$ induces a quasi-isometric embedding

$$
\text { Cayley }\left(g P g^{-1}, \mathcal{T}\right) \rightarrow \operatorname{Cayley}(G, \mathcal{S})
$$

The next result states that a (sufficiently large) saturation of a geodesic is quasiconvex. Furthermore, neighborhoods of saturations are also quasiconvex.

Theorem 8.4 [13, Theorem 1.12(4)] Let $(G, \mathbb{P})$ be a relatively hyperbolic group with finite generating set $\mathcal{S}$. For each $\epsilon>0$, there is a constant $M=M(\epsilon)$ such that the following holds. Let $c$ be an $\epsilon$-quasigeodesic in Cayley $(G, \mathcal{S})$ and let $\widehat{c}$ be a geodesic in Cayley $(G, \mathcal{S} \cup \mathcal{P})$ with the same endpoints as $c$. If $\tilde{c}$ is any lift of $\hat{c}$ then $\tilde{c}$ lies in the $M$-neighborhood of the $M$-saturation of $c$ with respect to the metric $d_{\mathcal{S}}$.

Theorem 8.5 [13, Theorem 4.1] Suppose $(G, \mathbb{P})$ is relatively hyperbolic with finite generating set $\mathcal{S}$. For each $M<\infty$ there is a constant $\iota=\iota(M)<\infty$ so that for any two peripheral cosets $g P \neq g^{\prime} P^{\prime}$ we have

$$
\operatorname{diam}\left(\mathcal{N}_{M}(g P) \cap \mathcal{N}_{M}\left(g^{\prime} P^{\prime}\right)\right)<\iota
$$

with respect to the metric $d_{\mathcal{S}}$.

The preceding result has the following stronger form that restricts the possible interactions between two distinct peripheral cosets in a saturation of a quasigeodesic.

Proposition 8.6 [13, Lemma 8.11] Let $(G, \mathbb{P})$ be a relatively hyperbolic group with finite generating set $\mathcal{S}$. For each choice of positive constants $\epsilon, v$, and $\tau$, there is a constant $\eta_{0}=\eta_{0}(\epsilon, v, \tau)$ such that the following holds. Let $c$ be an $\epsilon$-quasigeodesic in Cayley $(G, \mathcal{S})$. Suppose $g P$ and $g^{\prime} P^{\prime}$ are distinct peripheral cosets in $\operatorname{Sat}_{v}(c)$. Then

$$
\mathcal{N}_{\tau}(g P) \cap \mathcal{N}_{\tau}\left(g^{\prime} P^{\prime}\right) \subseteq \mathcal{N}_{\eta_{0}}(c)
$$

with respect to the metric $d_{\mathcal{S}}$. 
Lemma 8.7 Let $(G, \mathbb{P})$ be a relatively hyperbolic group with finite generating set $\mathcal{S}$. Choose positive constants $\epsilon, \nu$, and $\tau$. Then there exists a constant $\eta_{1}=\eta_{1}(\epsilon, \nu, \tau)$ such that the following holds in the graph $\operatorname{Cayley}(G, \mathcal{S})$. Let $b$ be an $\epsilon$-quasigeodesic, and suppose $C$ is a connected subset of $\mathcal{N}_{\tau}\left(\operatorname{Sat}_{v}(c)\right)$. If $C$ does not intersect $\mathcal{N}_{\eta_{1}}(c)$, then $C$ intersects $\mathcal{N}_{\tau}(g P)$ for a unique peripheral coset $g P \subseteq \operatorname{Sat}_{v}(c)$. Furthermore, $C \subseteq \mathcal{N}_{\tau}(g P)$.

Proof By hypothesis, $C$ is contained in the following union of open sets:

$$
\mathcal{N}_{\tau}(c) \cup\left(\bigcup\left\{\mathcal{N}_{\tau}(g P) \mid g P \subseteq \operatorname{Sat}_{v}(c)\right\}\right)
$$

Assume that $C$ does not intersect $\mathcal{N}_{\tau}(c)$. If $C$ intersects $\mathcal{N}_{\tau}(g P) \cap \mathcal{N}_{\tau}\left(g^{\prime} P^{\prime}\right)$ for distinct peripheral cosets $g P$ and $g^{\prime} P^{\prime}$ contained in $\operatorname{Sat}_{v}(c)$, then Proposition 8.6 gives a constant $\eta_{0}=\eta_{0}(\epsilon, \nu, \tau)$ such that $C$ intersects $\mathcal{N}_{\eta_{0}}(c)$. Otherwise, $C$ intersects $\mathcal{N}_{\tau}(c)$ for a unique peripheral coset $g P \subseteq \operatorname{Sat}_{v}(c)$, and thus $C \subseteq \mathcal{N}_{\tau}(g P)$.

Lemma 8.8 Let $(G, \mathbb{P})$ be a relatively hyperbolic group with a finite generating set $\mathcal{S}$. For each $\epsilon>0$ there is a constant $A_{0}=A_{0}(\epsilon)$ such that the following holds. Let $c$ be an $\epsilon$-quasigeodesic segment in $\operatorname{Cayley}(G, \mathcal{S})$, and let $\widehat{c}$ be a geodesic segment in Cayley $(G, \mathcal{S} \cup \mathcal{P})$. Suppose $c$ and $\hat{c}$ have the same endpoints in $G$. Then each vertex of $\hat{c}$ lies in the $A_{0}$-neighborhood of some vertex of $c$ with respect to the metric $d_{\mathcal{S}}$.

Proof Let $\tilde{c}$ be a lift of $\hat{c}$ to $\operatorname{Cayley}(G, \mathcal{S})$. By Theorem 8.4, $\tilde{c}$ lies in the $M-$ neighborhood of the $M$-saturation of $c$ for some $M$ depending only on $\epsilon$ and the generating set $\mathcal{S}$. Let $\iota=\iota(M)$ be the constant given by Theorem 8.5. Let $v$ be a vertex of $\hat{c}$. If $v$ is within an $\mathcal{S}$-distance $2 \iota M$ of an endpoint of $\tilde{c}$, then we are done. Otherwise let $\gamma$ be the subpath of $\tilde{c}$ of $\mathcal{S}$-length $4 \iota M$ centered at $v$. Since $\gamma$ is connected, either $\gamma$ lies in the $M$-neighborhood of some coset $g P \subseteq \operatorname{Sat}_{M}(c)$, or $\gamma$ intersects $\mathcal{N}_{\eta_{1}}(c)$, where $\eta_{1}=\eta_{1}(1, M, M)$ is given by Lemma 8.7.

We will show that $\gamma$ intersects $\mathcal{N}_{\eta_{1}}(c)$. Assume by way of contradiction that $\gamma \subseteq$ $\mathcal{N}_{M}(g P)$ for $g P \subseteq \operatorname{Sat}_{M}(c)$. It follows that the endpoints $x$ and $y$ of $\gamma$ are connected by a relative geodesic $\hat{\gamma}$ passing through $v$, each of whose vertices lies within an $\mathcal{S}$ distance $M$ of $g P$. Evidently $\hat{\gamma}$ contains at most $2 M+1$ edges. If an edge $e$ of $\hat{\gamma}$ does not have both endpoints in $g P$, then its endpoints are separated by an $\mathcal{S}$-distance less than $\iota$ by Theorem 8.5. At most one edge of $\hat{\gamma}$ has both endpoints in $g P$. Without loss of generality, we may assume the subpath of $\hat{\gamma}$ from $x$ to $v$ does not contain such an edge. Since $d_{\mathcal{S} \cup \mathcal{P}}(x, v) \leq 2 M$, it follows that $d_{\mathcal{S}}(x, v)<2 \iota M$, contradicting our choice of path $\gamma$.

Thus $\gamma$ intersects $\mathcal{N}_{\eta_{1}}\left(c^{\prime}\right)$ as desired. It follows that $v$ is within an $\mathcal{S}$-distance $2 \iota M+\eta_{1}$ of $c$, completing the proof. 
Definition 8.9 Let $c$ be a geodesic of Cayley $(G, \mathcal{S})$, and let $\epsilon, R$ be positive constants. A point $x \in c$ is $(\epsilon, R)$-deep in a peripheral left coset $g P$ (with respect to $c$ ) if $x$ is not within a distance $R$ of an endpoint of $c$ and $B(x, R) \cap c$ lies in $\mathcal{N}_{\epsilon}(g P)$. If $x$ is not $(\epsilon, R)$-deep in any peripheral left coset $g P$ then $x$ is an $(\epsilon, R)$-transition point of $c$.

Lemma 8.10 Let $(G, \mathcal{S})$ be relatively hyperbolic with finite generating set $\mathcal{S}$. For each $\epsilon$ there is a constant $R=R(\epsilon)$ such that the following holds. Let $c$ be any geodesic of Cayley $(G, \mathcal{S})$, and let $\bar{c}$ be a connected component of the set of all $(\epsilon, R)-$ deep points of $c$. Then there is a peripheral left coset $g P$ such that each $x \in c$ is $(\epsilon, R)$-deep in $g P$ and is not $(\epsilon, R)$-deep in any other peripheral left coset.

Proof Let $g P$ and $g^{\prime} P^{\prime}$ be distinct left cosets of peripheral subgroups. Then $\mathcal{N}_{\epsilon}(g P) \cap \mathcal{N}_{\epsilon}\left(g^{\prime} P^{\prime}\right)$. has diameter less than $R$, where $R:=\iota(\epsilon)$ is the constant given by Theorem 8.5.

Let $c_{g P}:=c \cap \mathcal{N}_{\epsilon}(g P)$. (Note that this set might not be connected.) Deleting the points that are within a distance $R$ of an endpoint of $c_{g P}$ gives precisely the set of points of $c$ that are $(\epsilon, R)$-deep in $g P$. Evidently the intersection $c_{g P} \cap c_{g^{\prime}} P^{\prime}$ has diameter at most $R$ since it lies in $\mathcal{N}_{\epsilon}(g P) \cap \mathcal{N}_{\epsilon}\left(g^{\prime} P^{\prime}\right)$. It is clear that if $x$ is $(\epsilon, R)$-deep in $g P$ and $y$ is $(\epsilon, R)$-deep in $g^{\prime} P^{\prime}$ then $d(x, y) \geq R$. In particular, within each component $\bar{c}$ of the set of $(\epsilon, R)$-deep points, every point is $(\epsilon, R)$-deep in a unique peripheral left coset $g P$ that depends only on the choice of component $\bar{c}$.

If $\bar{c}$ is a component of the $(\epsilon, R)$-deep points as above, we say that $\bar{c}$ is $(\epsilon, R)-$ deep in $g P$, where $g P$ is the unique peripheral left coset associated to every point of $\bar{c}$.

Lemma 8.11 For each $\epsilon, L>0$, there is a constant $\eta=\eta(\epsilon, L)$ so that the following holds. Suppose $c$ is a geodesic in $\operatorname{Cayley}(G, \mathcal{S})$ that lies in $\mathcal{N}_{\epsilon}(g P)$ for some left coset of a peripheral subgroup $P \in \mathbb{P}$. Suppose $\widehat{c}$ is a geodesic in Cayley $(G, \mathcal{S} \cup \mathcal{P})$ such that each point of $c$ is within an $\mathcal{S}$-distance $L$ of some vertex of $\widehat{c}$. Then $c$ has length at most $\eta$.

Proof Let $v_{0}, \ldots, v_{n}$ be the vertices of $\widehat{c}$. Without loss of generality we can assume that $v_{0}$ and $v_{n}$ both lie within an $\mathcal{S}$-distance $L$ of $c$. (If not, then we first shorten $\widehat{c}$ so that this condition holds.) Let $c_{i} \subseteq c$ be the set $\mathcal{N}_{L}\left(v_{i}\right) \cap c$. Then $c$ is covered by the sets $c_{0}, \ldots, c_{n}$, each of which has diameter at most $2 L$.

In order to bound the length of $c$, it suffices to bound the integer $n$ in terms of $\epsilon$ and $L$. But $v_{0}$ and $v_{n}$ are each within an $\mathcal{S}$-distance $\epsilon+L$ of a vertex of $g P$. Thus $d_{\mathcal{S} \cup \mathcal{P}}\left(v_{0}, v_{n}\right) \leq 2(\epsilon+L)+1$. In particular, $n$ is at most $2(\epsilon+L)+1$, completing the proof. 
Definition 8.12 Let $c$ be a geodesic in a space $X$. If $c_{0}$ is any subset of $c$, then the hull of $c_{0}$ in $c$, denoted Hull $l_{c}\left(c_{0}\right)$ is the smallest connected subspace of $c$ containing $c_{0}$.

Proposition 8.13 Let $(G, \mathbb{P})$ be relatively hyperbolic with a finite generating set $\mathcal{S}$. There exist constants $\epsilon, R$, and $L$ such that the following holds. Let $c$ be any geodesic of Cayley $(G, \mathcal{S})$ with endpoints in $G$, and let $\hat{c}$ be a geodesic of Cayley $(G, \mathcal{S} \cup \mathcal{P})$ with the same endpoints as $c$. Then in the metric $d_{\mathcal{S}}$, the set of vertices of $\hat{c}$ is at a Hausdorff distance at most $L$ from the set of $(\epsilon, R)$-transition points of $c$. Furthermore, the constants $\epsilon$ and $R$ satisfy the conclusion of Lemma 8.10

Proof Let $A_{0}=A_{0}(1)$ be the constant given by Lemma 8.8. Then each vertex of $\hat{c}$ is within a distance $A_{0}$ of $c$. For each vertex $v$ of $\widehat{c}$, let

$$
c_{v}:=\operatorname{Hull}_{c}\left(c \cap B\left(v, A_{0}\right)\right) .
$$

For each edge $e$ of $\widehat{c}$ with endpoints $v$ and $w$, let

$$
c_{e}:=\operatorname{Hull}_{c}\left(c_{v} \cup c_{w}\right) .
$$

Then $c$ is covered by the sets $c_{e}$ for all edges $e$ of $\hat{c}$.

If $e$ is labelled by an edge of $\mathcal{S}$, then $c_{e}$ has length at most $2 A_{0}+1$. Thus every point of $c_{e}$ lies within a distance $2 A_{0}+1$ of $c \cap B\left(v, A_{0}\right)$, and hence lies within a distance $3 A_{0}+1$ of $v$, where $v$ is a vertex incident to $e$.

On the other hand, if $e$ is labelled by an edge of $\mathcal{P}$ then the endpoints of $e$ lie in the same left coset $g P$ of some peripheral subgroup. Thus $c \cap B\left(v, A_{0}\right)$ lies in the $A_{0}-$ neighborhood of $g P$ for each endpoint $v$ of $e$. As each point of $c_{e}$ lies between two such points, it follows that $c_{e}$ lies in the $A_{1}$-neighborhood of $g P$ where $A_{1}=A_{1}\left(A_{0}\right)$ is the constant given by Theorem 8.1. Let $R:=R\left(A_{1}\right)$ be the constant given by Lemma 8.10. The points of $c_{e}$ within a distance $R$ of the endpoints of $c_{e}$ are also within a distance $A_{0}+R$ of the vertices of $\hat{c}$. All other points of $c_{e}$ must be $\left(A_{1}, R\right)$-deep points of $c$. In particular, we have shown that the $\left(A_{1}, R\right)$-transition points of $c$ each lie within a distance $3 A_{0}+R+1$ of the set of vertices of $\hat{c}$.

In order to complete the proof, we need to bound the distance from an arbitrary vertex of $\hat{c}$ to the set of $\left(A_{1}, R\right)$-transition points of $c$. Choose a vertex $v \in \widehat{c}$. If $c_{v}$ contains an $\left(A_{1}, R\right)$-transition point, then we are done, since $c_{v}$ has length at most $2 A_{0}$ and intersects $B\left(v, A_{0}\right)$. It suffices to assume that $c_{v}$ is contained in some $\left(A_{1}, R\right)$-deep component $\bar{c}$ of $c$ that is deep in a peripheral left coset $g P$. By Lemma 8.10, each endpoint of $\bar{c}$ is an $\left(A_{1}, R\right)$-transition point of $c$, so we need only bound the distance from $c_{v}$ to an endpoint of $\widehat{c}$. 
Since $\hat{c}$ is a geodesic of $\operatorname{Cayley}(G, \mathcal{S} \cup \mathcal{P})$, it contains at most one edge $e_{0}$ whose endpoints both lie in the coset $g P$. Choose an edge path $e_{1}, \ldots, e_{k}$ in $\widehat{c}$ such that $v$ is the initial vertex of $e_{1}$, such that for $i=1, \ldots, k-1$ we have $c_{e_{i}} \subseteq \bar{c}$, and such that $c_{k}$ contains an endpoint of $\bar{c}$. Since $\bar{c}$ has two endpoints, we can also choose $e_{1}, \ldots, e_{k}$ so that none of the edges is equal to $e_{0}$ (if there is such an edge $e_{0}$ ).

Let $w$ denote the common endpoint of $e_{k-1}$ and $e_{k}$. Both $v$ and $w$ lie within a $d_{\mathcal{S}}$-distance $A_{0}+A_{1}$ of $g P$, so $d_{\mathcal{S} \cup \mathcal{P}}(v, w)<2 A_{0}+2 A_{1}+1$. Therefore $k-1 \leq$ $2 A_{0}+2 A_{1}+1$. Suppose $i=1, \ldots, k-1$. If $e_{i}$ is labeled by an element of $\mathcal{S}$ then, as noted above, $c_{e_{i}}$ has length at most $2 A_{0}+1$. On the other hand, if $e_{i}$ is labelled by an element of $\mathcal{P}$, then the endpoints of $e_{i}$ lie in a peripheral left coset $g^{\prime} P^{\prime} \neq g P$. Since $c_{e_{i}} \subseteq \bar{c}$ cannot contain any points that are $\left(A_{1}, R\right)$-deep in $g^{\prime} P^{\prime}$, it follows that $c_{e_{i}}$ has length less than $2 R$. So we have an upper bound on the $d_{\mathcal{S}}$-distance from $v$ to $w$.

Now consider the last edge $e_{k}$ of our edge path, which contains an endpoint of $\bar{c}$. If $e_{k}$ is labelled by an edge of $\mathcal{S}$, then we are done, since the $d_{\mathcal{S}}$-distance from $w$ to this endpoint is bounded above by the length of $e_{k}$, which is at most $2 A_{0}+1$. If $e_{k}$ is labelled by an edge of $\mathcal{P}$, then $\bar{c}$ can intersect $c_{e_{k}}$ in a subsegment of length at most $R$, so $w$ is within a $d_{\mathcal{S}}$-distance $R$ of an endpoint of $\bar{c}$, completing the proof.

It is worth noting the following corollary, which could also be derived directly from the work of Druț-Sapir [13]. The corollary deals with only the geometry of the finite generating set $\mathcal{S}$.

Corollary 8.14 Let $(G, \mathbb{P})$ be a relatively hyperbolic group with finite generating set $\mathcal{S}$. Then there exist constants $\epsilon, R, M$ such that $\epsilon$ and $R$ satisfy the conclusion of Lemma 8.10 and such that the following holds. Let $c$ and $c^{\prime}$ be two geodesics in Cayley $(G, \mathcal{S})$ with the same endpoints in $G$. Then the set of $(\epsilon, R)$-transition points of $c$ and the set of $(\epsilon, R)$-transition points of $c^{\prime}$ are at a Hausdorff distance at most $M$.

We also recover the following result due to Osin [27, Proposition 3.15]. We remark that if $\mathcal{S}$ is a relative generating set rather than a generating set, the result still holds and can be derived as a corollary of Osin [28, Proposition 3.2].

Corollary 8.15 Let $(G, \mathbb{P})$ be a relatively hyperbolic group with finite generating set $\mathcal{S}$. Then there exists a constant $N$ such that if $c$ and $c^{\prime}$ are two geodesics in Cayley $(G, \mathcal{S} \cup \mathcal{P})$ with the same endpoints in $G$, then the set of vertices of $c$ and the set of vertices of $c^{\prime}$ are within a Hausdorff distance $N$ in the metric $d_{\mathcal{S}}$. 
Finally, we deduce the following corollary describing relatively quasiconvex subgroups of $G$ using only the geometry of Cayley $(G, \mathcal{S})$.

Corollary 8.16 Let $(G, \mathbb{P})$ be relatively hyperbolic with finite generating set $\mathcal{S}$. There are $\epsilon, R$ satisfying the conclusion of Lemma 8.10 such that the following holds. Let $H$ be a subgroup of $G$. Then $H$ is relatively quasiconvex if and only if there is a constant $\kappa$ such that for each geodesic $c$ in $\operatorname{Cayley}(G, \mathcal{S})$ joining points of $H$, the set of $(\epsilon, R)$-transition points of $c$ lies in the $\kappa$-neighborhood of $H$ (with respect to the metric $\left.d_{\mathcal{S}}\right)$.

Proof A subgroup $H \leq G$ is relatively quasiconvex if and only if it satisfies (QC-5) with respect to the generating set $\mathcal{S}$ and the proper left invariant metric $d_{\mathcal{S}}$. The corollary now follows immediately from Proposition 8.13.

\section{Applications of relative quasiconvexity}

The present section is a collection of various basic properties of relatively quasiconvex subgroups. In particular, we prove Theorems 1.2 and 1.5 and Corollary 1.3. We also briefly examine strongly relatively quasiconvex subgroups, which were introduced by Osin in [27].

The following theorem states that relatively quasiconvex subgroups are relatively hyperbolic, with the obvious peripheral structure.

Theorem 9.1 (Relatively quasiconvex $\Rightarrow$ relatively hyperbolic) Let $(G, \mathbb{P})$ be relatively hyperbolic, and let $H \leq G$ be a relatively quasiconvex subgroup. Consider the following collection of subgroups of $H$ :

$$
\overline{\mathbb{O}}:=\left\{H \cap g P g^{-1} \mid g \in G, P \in \mathbb{P} \text {, and } H \cap g P g^{-1} \text { is infinite }\right\} .
$$

Then the elements of $\overline{\mathbb{O}}$ lie in only finitely many conjugacy classes in $H$. Furthermore, if $\mathbb{O}$ is a set of representatives of these conjugacy classes then $(H, \mathbb{O})$ is relatively hyperbolic.

The peripheral structure $\mathbb{O}$ constructed above is referred to as an induced peripheral structure on $H$ coming from $(G, \mathbb{P})$. Note that the only ambiguity in the construction of $\mathbb{O}$ is the choice of representatives of the conjugacy classes from $\overline{\mathbb{O}}$.

Proof We give a dynamical proof based on geometrically finite convergence group actions. Let $(G, \mathbb{P})$ act geometrically finitely on a compactum $M$. Since $H$ is $(\mathrm{QC}-1)$, the induced action of $H$ on $\Lambda(H) \subseteq M$ is geometrically finite. 
By a result of Tukia, a point cannot be both a conical limit point and a parabolic point for the same convergence action [35, Theorem 3A]. Every conical limit point for $H$ acting on $\Lambda(H) \subseteq M$ is a conical limit point for $H$ acting on $M$, and hence also a conical limit point for $G$ acting on $M$. On the other hand, every parabolic point for $H$ acting on $\Lambda(H)$ is clearly a parabolic point for $G$ acting on $M$. Thus the parabolic points of $H$ are precisely the parabolic points of $G$ that lie in $\Lambda(H)$.

Consequently the set $\overline{\mathbb{O}}$ is the set of maximal parabolic subgroups for the action of $H$ on $\Lambda(H)$. Tukia has shown that a geometrically finite group action has only finitely many conjugacy classes of maximal parabolic subgroups [35, Theorem 1B]. Thus $(H, \mathbb{O})$ satisfies $(\mathrm{RH}-1)$.

A group $G$ is slender if every subgroup $H \leq G$ is finitely generated. The following corollary is an easy consequence of the preceding theorem.

Corollary 9.2 Let $(G, \mathbb{P})$ be relatively hyperbolic. The following conditions are equivalent:

(1) Every $P \in \mathbb{P}$ is slender.

(2) Every relatively quasiconvex subgroup $H$ of $(G, \mathbb{P})$ is finitely generated.

Proof $(1) \Rightarrow$ (2) If $H$ is relatively quasiconvex, then it is relatively hyperbolic with respect to subgroups of the $P \in \mathbb{P}$. If each such $P$ is slender, then $H$ is relatively hyperbolic with respect to finitely generated groups. In particular $H$ is finitely generated relative to finitely many finitely generated subgroups. Thus $H$ is finitely generated.

(2) $\Rightarrow$ (1) This holds since every subgroup $H$ of a peripheral subgroup $P \in \mathbb{P}$ is relatively quasiconvex.

Since a relatively quasiconvex subgroup $H$ of a relatively hyperbolic group $G$ is itself relatively hyperbolic, we could in principle consider nested sequences $H_{0} \leq H_{1} \leq$ $\cdots \leq H_{\ell}=G$ such that $H_{i}$ is relatively quasiconvex in $H_{i+1}$. The following corollary shows that any subgroup $H_{0}$ produced in this manner is relatively quasiconvex in the original group $G$. Thus no new subgroups arise in this manner.

Corollary 9.3 (Nested relative quasiconvexity) Suppose $(G, \mathbb{P})$ is relatively hyperbolic, and $K \leq G$ is relatively quasiconvex in $(G, \mathbb{P})$ with induced peripheral structure $\mathbb{O}$. Suppose $H \leq K$. Then $H$ is relatively quasiconvex in $(K, \mathbb{O})$ if and only if $H$ is relatively quasiconvex in $(G, \mathbb{P})$. 
Proof Let $(G, \mathbb{P})$ act geometrically finitely on a compactum $M$. Then the limit set $\Lambda(H)$ for the action of $H$ on $M$ is the same as the limit set for the restricted action of $H$ on $\Lambda(K)$. The property of geometrical finiteness of the action of $H$ on $\Lambda(H)$ is intrinsic to $\Lambda(H)$. Thus $H$ satisfies $(\mathrm{QC}-1)$ as a subgroup of $(G, \mathbb{P})$ if and only if $H$ satisfies $(\mathrm{QC}-1)$ as a subgroup of $(K, \mathbb{O})$.

Using Lemma 8.8, the proof of Theorem 1.5 is straightforward.

Proof of Theorem 1.5 Since Cayley $(H, \mathcal{T})$ is a geodesic space, it follows that each pair of points in $H$ is connected in Cayley $(G, \mathcal{S})$ by an $\epsilon$-quasigeodesic that lies in the $\epsilon$-neighborhood of $H$. Let $c$ be such a quasigeodesic. By Lemma 8.8, if $\widehat{c}$ is any geodesic of Cayley $(G, \mathcal{S} \cup \mathcal{P})$ with the same endpoints as $c$, then each vertex $v$ of $\hat{c}$ lies within a $d_{\mathcal{S}}$-distance $A_{0}$ of $c$, where $A_{0}=A_{0}(\epsilon)$. Consequently, $v$ is within a $d_{\mathcal{S}}$-distance $A_{0}+\epsilon$ of some point of $H$, establishing that $H$ satisfies definition $(\mathrm{QC}-5)$ with respect to the generating set $\mathcal{S}$ and the proper, left invariant metric $d_{\mathcal{S}}$.

The following result roughly states that an element of a group that lies close to two cosets $x H$ and $y K$ also lies near the intersection $x H x^{-1} \cap y K^{-1}$.

Proposition 9.4 Let $G$ have a proper, left invariant metric $d$, and suppose $x H$ and $y K$ are arbitrary left cosets of subgroups of $G$. For each constant $L$ there is a constant $L^{\prime}=L^{\prime}(G, d, x H, y K)$ so that in the metric space $(G, d)$ we have

$$
\mathcal{N}_{L}(x H) \cap \mathcal{N}_{L}(y K) \subseteq \mathcal{N}_{L^{\prime}}\left(x H x^{-1} \cap y K y^{-1}\right) .
$$

Proof If there is no such $L^{\prime}$, then there is a sequence $\left(z_{i}\right)$ in $G$ so that $z_{i}$ is in the $L$-neighborhood of both $x H$ and $y K$, but $i<d\left(z_{i}, x H x^{-1} \cap y K y^{-1}\right)$ for each $i$. It follows that $z_{i}=x h_{i} p_{i}=y k_{i} q_{i}$ for some $h_{i} \in H, k_{i} \in K$ and $p_{i}, q_{i} \in G$ with $d\left(1, p_{i}\right)$ and $d\left(1, q_{i}\right)$ both less than $L$. Since the ball of radius $L$ in $(G, d)$ is finite, we can pass to a subsequence in which $p_{i}$ and $q_{i}$ are constants $p$ and $q$. Then for each $i$ we can express $z_{i}=x h_{i} p=y k_{i} q$. Therefore

$$
z_{i} z_{1}^{-1}=x h_{i} h_{1}^{-1} x^{-1}=y k_{i} k_{1}^{-1} y^{-1} \in x H x^{-1} \cap y K y^{-1} .
$$

It follows that the distance between $z_{i}$ and $x H x^{-1} \cap y K y^{-1}$ is at most $d\left(1, z_{1}\right)$ for all $i$, contradicting our choice of $\left(x_{i}\right)$.

Corollary 9.5 Let $H$ and $K$ be relatively quasiconvex subgroups of a relatively hyperbolic group $(G, \mathbb{P})$. Then $H \cap K$ is relatively quasiconvex in $(G, \mathbb{P})$. 
Proof Choose a finite relative generating set $\mathcal{S}$ for $(G, \mathbb{P})$ and a proper, left invariant metric $d$ for $G$. Consider the Cayley graph $\bar{\Gamma}:=\operatorname{Cayley}(G, \mathcal{S} \cup \mathcal{P})$. If $c$ is a geodesic in $\bar{\Gamma}$ with both endpoints in $H \cap K$, then each vertex $v$ of $c$ lies within a uniformly bounded $d$-distance of both $H$ and $K$ by (QC-5). Therefore, by Proposition 9.4 we also have a uniform bound on the distance $d(v, H \cap K)$. Thus $H \cap K$ satisfies (QC-5) as well.

Theorem 9.1 and Corollary 9.5 together complete the proof of Theorem 1.2. The proof of Corollary 1.3 is now immediate.

Proof of Corollary 1.3 Let $H$ be a subgroup of a geometrically finite group $G \leq$ Isom $(X)$, where $X$ is a finite volume manifold with pinched negative curvature. The first assertion of the Corollary follows from Definition (QC-1) for relative quasiconvexity. The second assertion is an immediate consequence of Corollary 9.5.

The notion of a strongly relatively quasiconvex subgroup was introduced by Osin in [27, Section 4.2] in the special case that $G$ is finitely generated. Osin's results about such subgroups extend easily to the general case. We give the definition and then list several basic results about strongly relatively hyperbolic subgroups.

Definition 9.6 Let $(G, \mathbb{P})$ be relatively hyperbolic. A subgroup $H \leq G$ is strongly relatively quasiconvex if $H$ is relatively quasiconvex and the induced peripheral structure $\mathbb{O}$ on $H$ is empty. In other words, the subgroup $H \cap g P g^{-1}$ is finite for all $g \in G$ and $P \in \mathbb{P}$.

Theorem 9.7 (See Theorem 4.16 of [27].) If $H$ is strongly relatively quasiconvex in $(G, \mathbb{P})$ then $H$ is finitely generated and word hyperbolic.

Proof The result is immediate, since $(H, \varnothing)$ is relatively hyperbolic.

We also obtain the following result, proved by Osin in the case that $G$ is finitely generated [27, Proposition 4.18]. (We remark that Osin erroneously claims to have proven Corollary 9.5, although his proof actually gives the result below.)

Theorem 9.8 Let $H$ and $K$ be subgroups of a relatively hyperbolic group $(G, \mathbb{P})$. If $H$ is strongly relatively quasiconvex and $K$ is relatively quasiconvex, then $H \cap K$ is strongly relatively quasiconvex.

Proof Since $H \cap K$ is a relatively quasiconvex subgroup of $(H, \varnothing)$, its induced peripheral structure must be empty. 
The following theorem characterizes strongly relatively quasiconvex subgroups in several ways.

Theorem 9.9 Let $(G, \mathbb{P})$ be a relatively hyperbolic group with finite relative generating set $\mathcal{S}$. Choose a cusp uniform action of $(G, \mathbb{P})$ on a $\delta$-hyperbolic space $(X, \rho)$. If $H$ is a subgroup of $G$, then the following are equivalent.

(1) $H$ is strongly relatively quasiconvex in $(G, \mathbb{P})$.

(2) $H$ acts on $\Lambda H \subseteq \partial X$ as a uniform convergence group; ie, the action of $H$ on the space of distinct triples of points of $\Lambda H$ is proper and cocompact.

(3) The action of $H$ on $\Lambda H \subseteq \partial X$ is a convergence group action, and every point of $\Lambda H$ is a conical limit.

(4) Either $H$ is finite, or the action of $H$ on join $(\Lambda X) \subseteq X$ is cocompact.

(5) For each basepoint $x \in X$ the orbit $H x \subseteq X$ is quasiconvex.

(6) $H$ is generated by a finite set $\mathcal{T}$ and for each basepoint $x \in X$ the orbit map $h \mapsto h(x)$ induces a quasi-isometric embedding

$$
\operatorname{Cayley}(H, \mathcal{T}) \rightarrow(X, \rho) \text {. }
$$

(7) $H$ is generated by a finite set $\mathcal{T}$ and the inclusion $H \hookrightarrow G$ induces a quasiisometric embedding

$$
\operatorname{Cayley}(H, \mathcal{T}) \rightarrow \operatorname{Cayley}(G, \mathcal{S} \cup \mathcal{P}) .
$$

Proof The equivalence of (2) and (3) for convergence group actions has been proved by Bowditch and also by Tukia [6; 35]. It is clear that (3) is simply definition (QC-1) with no parabolic subgroups. Similarly, (4) is simply (QC-2) with no parabolic subgroups. Thus the first four conditions are equivalent.

(4) $\Leftrightarrow(5)$ Suppose $H$ acts cocompactly on join $(\Lambda H)$. Choose $x \in \operatorname{join}(\Lambda H)$. Since join $(\Lambda H)$ is quasiconvex, it is clear that any geodesic joining points of $H x$ lies near $H x$.

Conversely, suppose $H x$ is quasiconvex. As in the proof of Proposition 7.6, each geodesic connecting points of $H x$ lies near $H x$, so that same holds for bi-infinite geodesics obtained as limits of such geodesics. Every point of $\Lambda H$ is a limit point of $H x$ by definition. Thus each pair in $\Lambda H$ is joined by such a geodesic $c$. Any other geodesic $c^{\prime}$ joining the same pair is at a Hausdorff distance $2 \delta$ from $c$. The set join $(\Lambda H)$ is the union of all such lines $c^{\prime}$, so join $(\Lambda H)$ lies in a uniformly bounded neighborhood of $H x$. In other words, $H$ acts cocompactly on join $(\Lambda H)$. 
(5) $\Leftrightarrow$ (6) This equivalence is elementary and well-known for subgroups $H$ of a word hyperbolic group $H^{\prime}$ by work of Short. Replace $H^{\prime}$ with $X$ to prove the desired result.

(1) $\Leftrightarrow(7)$ This equivalence is proved by Osin in the case that $\mathcal{S}$ generates $G$ [27, Theorem 4.13]. However Osin's proof never uses this hypothesis, so it extends without change to the present setting once we replace $d_{\mathcal{S}}$ with a proper, left invariant metric $d$ on $G$. (The forward implication also follows from Theorem 10.1 below.)

\section{Geometric properties of subgroup inclusion}

In this section, we examine the geometry of the inclusion $H \hookrightarrow G$ where $H$ is relatively quasiconvex in $(G, \mathbb{P})$. Theorem 10.1 shows that the relative Cayley graph of $H$ embeds quasi-isometrically into the relative Cayley graph of $G$. In Theorem 10.5 we compute the distortion of $H$ in $G$ in the case when $H$ and $G$ are both finitely generated. We finish the section with a proof of Corollary 1.6.

If $(G, \mathbb{P})$ is relatively hyperbolic, recall that $\mathcal{P}$ is the disjoint union

$$
\mathcal{P}:=\coprod_{P \in \mathbb{P}}(\widetilde{P}-\{1\}),
$$

where $\widetilde{P}$ is an abstract group isomorphic to $P$. If $H$ is relatively quasiconvex in $(G, \mathbb{P})$ with induced peripheral structure $\mathbb{O}$, then the set $\mathcal{O}$ is defined analogously to $\mathcal{P}$.

Theorem 10.1 Let $(G, \mathbb{P})$ be relatively hyperbolic, and let $H$ be a relatively quasiconvex subgroup with induced peripheral structure $\mathbb{O}$. Choose finite relative generating sets $\mathcal{S}$ and $\mathcal{T}$ for $(G, \mathbb{P})$ and $(H, \mathbb{O})$ respectively. Then the inclusion $H \hookrightarrow G$ induces a quasi-isometric embedding

$$
f:\left(H, d_{\mathcal{T} \cup \mathcal{O}}\right) \rightarrow\left(G, d_{\mathcal{S} \cup \mathcal{P}}\right) .
$$

In principle, one could prove the theorem by modifying Short's original proof that quasiconvex subgroups of a hyperbolic group are finitely generated and undistorted [30]. However, it seems more direct to use electric geometry since in that setting the quasiisometric embedding is geometrically obvious.

Proof Let $(G, \mathbb{P})$ have a cusp uniform action on $(X, \rho)$. Recall that $Y^{\prime}:=\operatorname{join}(\Lambda H) \subseteq$ $X$ is $\kappa$-quasiconvex for some $\kappa>0$. By $(\mathrm{QC}-2)$, we know that $(H, \mathbb{O})$ has a cusp uniform action on a geodesic space $Y$ that is $H$-equivariantly quasi-isometric to $Y^{\prime}$ endowed with the subspace metric $\rho$. By abuse of notation we will also use $\rho$ to refer to the metric on $Y$. 
Choose a truncated space $X-U$ for $G$ such that the only horoballs of $U$ meeting $Y^{\prime}$ are those centered at parabolic points of $H$. Pull $X-U$ back to $Y$ to get an induced truncated space $Y-V$ for $H$. Suppose the horoballs of $U$ and also of $V$ are pairwise separated by at least a distance $r$, where $r$ is given by Lemma 6.8. Let $\hat{\rho}$ denote the electric metric associated to $(X, U)$, and also the electric metric associated to $(Y, V)$.

For any semipolygonal relative geodesic $(\gamma, \alpha)$ in $(X, U)$ connecting two points of join $(\Lambda H)$, the path $\gamma$ is a quasigeodesic of $(X, \rho)$ by Lemma 6.8. Thus by the Morse Lemma and the quasiconvexity of join $(\Lambda H)$, we see that $\gamma$ lies in the $L$-neighborhood of join $(\Lambda H)$ for some $L$ depending only on $\kappa$ and the choice of $U$. It follows that $(Y, \hat{\rho})$ is quasi-isometric to join $(\Lambda H)$ with the electric metric $\hat{\rho}$ induced as a subspace of $X$. Therefore the map $Y \rightarrow X$ induces an $H$-equivariant quasi-isometric embedding $(Y, \hat{\rho}) \rightarrow(X, \hat{\rho})$.

Applying Proposition 7.9 to both $Y$ and $X$, gives equivariant quasi-isometries

$$
\left(H, d_{\mathcal{T} \cup \mathcal{O}}\right) \rightarrow(Y, \hat{\rho}) \quad \text { and } \quad\left(G, d_{\mathcal{S} \cup \mathcal{P}}\right) \rightarrow(X, \hat{\rho}) .
$$

Thus the inclusion $H \hookrightarrow G$ induces a quasi-isometric embedding

$$
f:\left(H, d_{\mathcal{T} \cup \mathcal{O}}\right) \rightarrow\left(G, d_{\mathcal{S} \cup \mathcal{P}}\right),
$$

completing the proof.

Two monotone functions $f, g:[0, \infty) \rightarrow[0, \infty)$ are said to be $\simeq$ equivalent if $f \preceq g$ and $g \preceq f$, where $f \preceq g$ means that there exists a constant $C>0$ such that

$$
f(r) \leq C g(C r+C)+C r+C
$$

for all $r \geq 0$. One extends this equivalence to functions $f: \mathbb{N} \rightarrow[0, \infty)$ by extending $f$ to be constant on each interval $[n, n+1)$.

Definition 10.2 (Distortion) If $G$ is a group with finite generating set $\mathcal{S}$ and $H$ is a subgroup with finite generating set $\mathcal{T}$, the distortion of $(H, \mathcal{T})$ in $(G, \mathcal{S})$ is the function

$$
\Delta_{H}^{G}(n):=\max \left\{|h|_{\mathcal{T}} \mid h \in H \text { and }|h|_{\mathcal{S}} \leq n\right\} .
$$

$\mathrm{Up}$ to $\simeq$ equivalence, this function does not depend on the choice of the finite generating sets $\mathcal{S}$ and $\mathcal{T}$. A subgroup $H \leq G$ is undistorted if and only if $\Delta_{H}^{G} \simeq n$.

Definition 10.3 A function $f: \mathbb{N} \rightarrow \mathbb{N}$ is superadditive if

$$
f(a+b) \geq f(a)+f(b) \text { for all } a, b \in \mathbb{N} .
$$


The superadditive closure of a function $f: \mathbb{N} \rightarrow \mathbb{N}$ is the smallest superadditive function $\bar{f}$ such that $\bar{f}(n) \geq f(n)$ for all $n \in \mathbb{N}$. The superadditive closure is given by the formula

$$
\bar{f}(n):=\max \left\{f\left(n_{1}\right)+\cdots+f\left(n_{r}\right) \mid r \geq 1 \text { and } n_{1}+\cdots+n_{r}=n\right\} .
$$

See Brick [8] and Guba-Sapir [21] for more information about superadditive functions.

The following lemma is useful when passing from the peripheral subgroups of $G$ to the induced peripheral subgroups of $H$.

Lemma 10.4 Let $(G, \mathbb{P})$ be relatively hyperbolic, and suppose $H$ is a relatively quasiconvex subgroup with induced peripheral structure $\mathbb{O}$. Fix a proper, left invariant metric $d$ on $G$. For each constant $L>0$ there are constants $D=D(L)$ and $L^{\prime}=L^{\prime}(L)$ such that the following holds.

Let $\mathcal{A}$ be the finite collection of peripheral left cosets $g P$ of $(G, \mathbb{P})$ such that $H \cap$ $g P g^{-1} \in \mathbb{O}$. Let $\mathcal{B}_{L}$ be the finite collection of peripheral left cosets $g P$ that intersect the ball $B(1, L)$ in $(G, d)$ such that $H \cap g P g^{-1}$ is finite.

Suppose $g^{\prime} P$ is a peripheral left coset of $(G, \mathbb{P})$ such that $d\left(H, g^{\prime} P\right)<L$. Then $g^{\prime} P=h g P$ for some $h \in H$ and some $g P \in \mathcal{A} \cup \mathcal{B}_{L}$, and

$$
\mathcal{N}_{L}(H) \cap \mathcal{N}_{L}\left(g^{\prime} P\right) \subseteq \mathcal{N}_{L^{\prime}}\left(h\left(H \cap g P g^{-1}\right)\right) .
$$

Furthermore, if $g P \in \mathcal{B}_{L}$ then

$$
\operatorname{diam}\left(\mathcal{N}_{L}(H) \cap \mathcal{N}_{L}\left(g^{\prime} P\right)\right)<D .
$$

Proof Since $d\left(H, g^{\prime} P\right)<L$, we can express $g^{\prime} P$ as $h_{0} g P$ for some $h_{0} \in H$ and some coset $g P$ intersecting $B(1, L)$ in $(G, d)$. If $H \cap g P g^{-1}$ is finite, then $g P \in \mathcal{B}_{L}$. In this case, we let $h:=h_{0}$.

On the other hand, if $H \cap g P g^{-1}$ is infinite, then it is conjugate in $H$ to a peripheral subgroup of $\mathbb{O}$. In other words, there is $h_{1} \in H$ such that $H \cap h_{1} g P g^{-1} h_{1}^{-1} \in \mathbb{O}$. Therefore $h_{1} g P=\left(h_{1} h_{0}^{-1}\right) g^{\prime} P \in \mathcal{A}$. In this case, let $h:=h_{1} h_{0}^{-1}$. In either case we have $g^{\prime} P=h g P$ for some $g P \in \mathcal{A} \cup \mathcal{B}_{L}$ as desired.

Since $\mathcal{A} \cup \mathcal{B}_{L}$ is finite, Proposition 9.4 gives is a constant $L^{\prime}=L^{\prime}(L)$ such that for every $g P \in \mathcal{A} \cup \mathcal{B}_{L}$ we have

$$
\mathcal{N}_{L}(H) \cap \mathcal{N}_{L}(g P) \subseteq \mathcal{N}_{L^{\prime}}\left(H \cap g P g^{-1}\right) .
$$

Translating this statement by $h$ gives

$$
\mathcal{N}_{L}(H) \cap \mathcal{N}_{L}\left(g^{\prime} P\right) \subseteq \mathcal{N}_{L^{\prime}}\left(h\left(H \cap g P g^{-1}\right)\right) .
$$


Since $\mathcal{B}_{L}$ is finite, there is some $D_{0}<\infty$ such that for each $g P \in \mathcal{B}_{L}$ we have

$$
\operatorname{diam}\left(H \cap g P g^{-1}\right)<D_{0} .
$$

If we let $D:=D_{0}+2 L^{\prime}$, then

$$
\operatorname{diam}\left(\mathcal{N}_{L}(H) \cap \mathcal{N}_{L}\left(g^{\prime} P\right)\right)<D
$$

whenever $g P \in \mathcal{B}_{L}$.

Theorem 10.5 Let $(G, \mathbb{P})$ be relatively hyperbolic with a finite generating set $\mathcal{S}$. Let $H$ be a relatively quasiconvex subgroup with a finite generating set $\mathcal{T}$. Let $\mathbb{O}$ denote the induced peripheral structure on $H$. For each $O=H \cap g P g^{-1} \in \mathbb{O}$ let $\delta_{O}$ denote the distortion of the finitely generated group $O$ in the finitely generated group $\mathrm{gPg}^{-1}$. Then the distortion of $H$ in $G$ satisfies

$$
f \preceq \Delta_{H}^{G} \preceq \bar{f}
$$

where

$$
f(n):=\max _{O \in \mathbb{O}} \delta_{O}(n)
$$

and $\bar{f}$ is the superadditive closure of $f$.

We remark that if $\mathbb{O}$ is empty (ie, $H$ is strongly relatively quasiconvex) then $f(n)$ is considered to be identically zero, which is $\simeq$ equivalent to a linear function. Thus in the case of a strongly relatively quasiconvex subgroup $H$, the preceding theorem implies that $H$ is undistorted in $G$.

The outline of the proof is inspired by Short's proof that quasiconvex subgroups of a finitely generated group are finitely generated and undistorted [30].

Proof First let us see that $f \preceq \Delta_{H}^{G}$. If $\mathbb{O}$ is empty, there is nothing to show. Thus it suffices to check that $\delta_{O} \preceq \Delta_{H}^{G}$ for each $O \in \mathbb{O}$. Choose finite generating sets $\mathcal{A}$, $\mathcal{B}, \mathcal{S}$ and $\mathcal{T}$ for $g \mathrm{Pg}^{-1}, O, G$ and $H$ respectively such that $\mathcal{A} \subseteq \mathcal{S}$. Choose $z \in O$ such that

$$
|z|_{\mathcal{A}} \leq n \quad \text { and } \quad|z|_{\mathcal{B}}=\delta_{O}(n) .
$$

Since the distortion of $O$ in $H$ is linear, we have

$$
|z|_{\mathcal{S}} \leq n \text { and }|z|_{\mathcal{T}}>\left(\Delta_{O}^{H}\right)^{-1}\left(\delta_{O}(n)-1\right) \simeq \delta_{O}(n) .
$$

Thus $\delta_{O} \preceq \Delta_{H}^{G}$.

We will now consider the less trivial inequality $\Delta_{H}^{G} \preceq \bar{f}$. Fix a finite generating set $\mathcal{S}$ for $G$, and let $\epsilon, R, \kappa$ be the constants given by Corollary 8.16. Suppose $|h|_{\mathcal{S}} \leq n$, and 
let $c$ be a geodesic in Cayley $(G, \mathcal{S})$ from 1 to $h$. Then the set of $(\epsilon, R)$-transition points of $c$ lies in the $\kappa$-neighborhood of $H$ in Cayley $(G, \mathcal{S})$.

The geodesic $c$ is a concatenation of paths $c_{0}, c_{1}^{\prime}, c_{1}, \ldots, c_{\ell}^{\prime}, c_{\ell}$ such that each $c_{i}$ is a component of the set of $(\epsilon, R)$-transition points of $c$, and each $c_{i}^{\prime}$ is a component of the set of $(\epsilon, R)$-deep points of $c$. Each path $c_{i}^{\prime}$ is $(\epsilon, R)$-deep in a unique peripheral left coset $g_{i}^{\prime} P_{i}$ by Lemma 8.10 .

We will assume without loss of generality that each $c_{i}$ and each $c_{i}^{\prime}$ is a sequence of edges. Increasing $R$ and $\kappa$ slightly, we can also assume that each $c_{i}$ contains at least one edge, so that $c$ has length at least $\ell+1$; in other words, $\ell+1 \leq n$.

Lemma 10.4 gives constants $L^{\prime}=L^{\prime}(\kappa+\epsilon)$ and $D=D(\kappa+\epsilon)$ and sets of cosets $\mathcal{A}$ and $\mathcal{B}_{\kappa+\epsilon}$ such that for each $i$ we have $g_{i}^{\prime} P_{i}=k_{i} g_{i} P_{i}$ for some $k_{i} \in H$ and some $g_{i} P_{i} \in \mathcal{A} \cup \mathcal{B}_{\kappa+\epsilon}$. Furthermore, if we let $O_{i}=H \cap g_{i} P_{i} g_{i}^{-1}$, then in $\left(G, d_{\mathcal{S}}\right)$ the endpoints of $c_{i}^{\prime}$ lie in $\mathcal{N}_{\kappa}(H) \cap \mathcal{N}_{\epsilon}\left(g_{i}^{\prime} P_{i}\right)$, which is a subset of $\mathcal{N}_{L^{\prime}}\left(k_{i} O_{i}\right)$. If $b_{i}$ is the label on the path $c_{i}^{\prime}$, then we can choose elements $u_{i}$ and $v_{i}$ in $G$ such that $\left|u_{i}\right|_{\mathcal{S}}$ and $\left|v_{i}\right|_{\mathcal{S}}$ are less than $L^{\prime}$ and such that $o_{i}:=u_{i} b_{i} v_{i}^{-1}$ is an element of $O_{i}$ as shown in Figure 1.

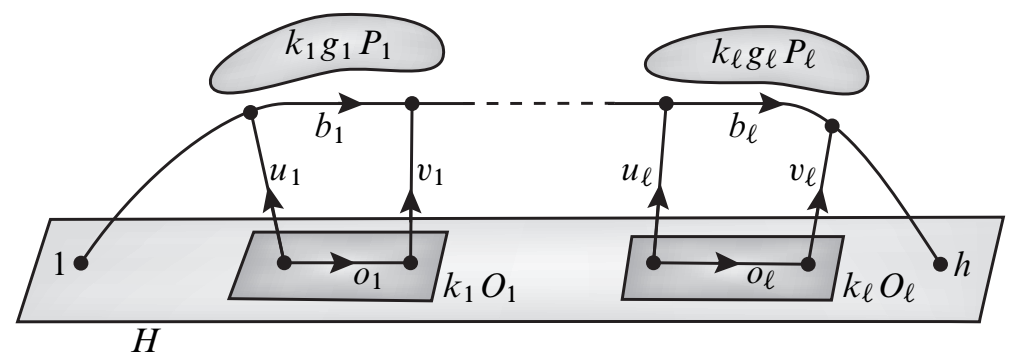

Figure 1: The elements $o_{i}$ lie in the peripheral subgroup $O_{i}$ of $H$.

Suppose the edges of $c_{i}$ are labelled by the sequence $a_{i, 1} \cdots a_{i, n_{i}}$ of elements of $\mathcal{S}$. Each vertex of $c_{i}$ lies within an $\mathcal{S}$-distance $\kappa$ of a vertex of $H$. Let $w_{0,0}=w_{\ell, n_{\ell}}=1$, and for each $i=1, \ldots, \ell$ let $w_{i-1, n_{i-1}}:=u_{i}$, and $w_{i, 0}:=v_{i}$. Then for each $j=$ $1, \ldots, n_{i}-1$ there exists $w_{i, j} \in G$ with $\left|w_{i, j}\right|_{\mathcal{S}}<\kappa$ such that whenever $j=1, \ldots, n_{i}$ the element $h_{i, j}:=w_{i, j-1} a_{i, j} w_{i, j}^{-1}$ lies in $H$ as illustrated in Figure 2.

We now have two decompositions of $h$. The first in $G$ corresponds to the labels along the geodesic $c$ :

$$
h=\left(a_{0,1} \cdots a_{0, n_{0}}\right) b_{1}\left(a_{1,1} \cdots a_{1, n_{1}}\right) \cdots b_{\ell}\left(a_{\ell, 1} \cdots a_{\ell, n_{\ell}}\right) .
$$

The second is a decomposition of $h$ in $H$, that "tracks close" to $c$ :

$$
h=\left(h_{0,1} \cdots h_{0, n_{0}}\right) o_{1}\left(h_{1,1} \cdots h_{1, n_{1}}\right) \cdots o_{\ell}\left(h_{\ell, 1} \cdots h_{\ell, n_{\ell}}\right) .
$$




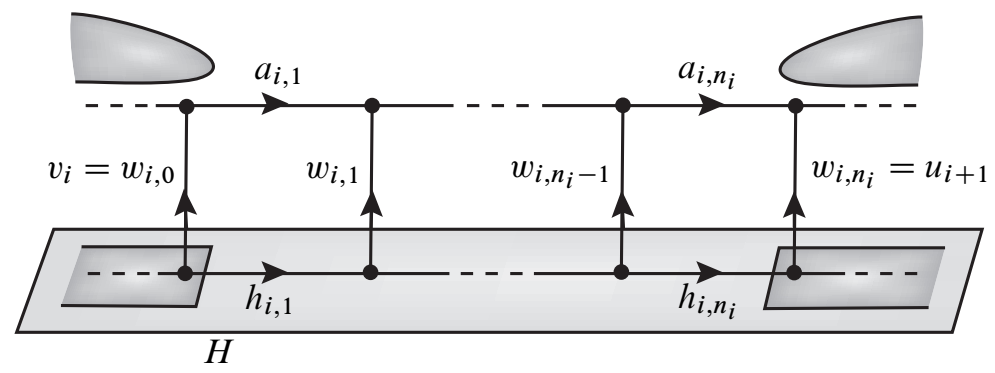

Figure 2: The elements $h_{i, j}$ lie in $H$.

Since $\left|w_{i, j}\right|_{\mathcal{S}}<\kappa+L^{\prime}$, we have $\left|h_{i, j}\right|_{\mathcal{S}}<2\left(\kappa+L^{\prime}\right)+1$. If $g_{i} P_{i} \in \mathcal{B}_{\kappa+\epsilon}$ for some $i$, then $\left|b_{i}\right|_{\mathcal{S}}<D$ by Lemma 10.4 , so that $\left|o_{i}\right|_{\mathcal{S}}<2 L^{\prime}+D$. Let $B$ denote the finite ball in $\left(H, d_{\mathcal{S}}\right)$ centered at 1 with radius $2\left(\kappa+L^{\prime}\right)+D+1$, and choose a finite generating set $\mathcal{T}$ for $H$ such that $B \subseteq \mathcal{T}$. Then we have

$$
\begin{aligned}
& |h|_{\mathcal{S}}=n_{0}+\left|b_{1}\right|_{\mathcal{S}}+n_{1}+\cdots+\left|b_{\ell}\right|_{\mathcal{S}}+n_{\ell} \leq n, \\
& |h|_{\mathcal{T}} \leq n_{0}+\left|o_{1}\right|_{\mathcal{T}}+n_{1}+\cdots+\left|o_{\ell}\right|_{\mathcal{T}}+n_{\ell} .
\end{aligned}
$$

If $g_{i} P_{i} \in \mathcal{B}_{\kappa+\epsilon}$ then $\left|o_{i}\right|_{\mathcal{T}} \leq 1$ by our choice of $\mathcal{T}$. On the other hand, if $g_{i} P_{i} \in \mathcal{A}$, then $O_{i} \in \mathbb{O}$. Since the distortions of $O_{i}$ in $H$ and of $g_{i} P_{i} g_{i}^{-1}$ in $G$ are linear, there is a constant $C$ depending only on $\mathbb{O}$ such that

$$
\begin{aligned}
\left|o_{i}\right|_{\mathcal{T}} & \leq C \delta_{O_{i}}\left(C\left|o_{i}\right|_{\mathcal{S}}+C\right)+C \\
& \leq C \delta_{O_{i}}\left(C\left(\left|u_{i}\right|_{\mathcal{S}}+\left|b_{i}\right|_{\mathcal{S}}+\left|v_{i}\right|_{\mathcal{S}}\right)+C\right)+C \\
& <C \delta_{O_{i}}\left(C\left|b_{i}\right|_{\mathcal{S}}+2 C L^{\prime}+C\right)+C \\
& \leq C \bar{f}\left(C\left|b_{i}\right|_{\mathcal{S}}+2 C L^{\prime}+C\right)+C .
\end{aligned}
$$

Using the superadditivity of $\bar{f}$ and the fact that $\ell<n$, we see that

$$
\begin{aligned}
|h|_{\mathcal{T}} & <\sum_{i=0}^{\ell} n_{i}+\sum_{i=1}^{\ell} C \bar{f}\left(C\left|b_{i}\right|_{\mathcal{S}}+2 C L^{\prime}+C\right)+C \\
& \leq n+C \bar{f}\left(\sum_{i=1}^{\ell} C\left|b_{i}\right|_{\mathcal{S}}+2 C L^{\prime}+C\right)+C \ell \\
& \leq n+C \bar{f}\left(C n+\left(2 C L^{\prime}+C\right) \ell\right)+C \ell \\
& \leq n+C \bar{f}\left(\left(2 C+2 C L^{\prime}\right) n\right)+C n .
\end{aligned}
$$

Thus $\Delta_{H}^{G} \preceq \bar{f}$ as desired. 
We will now use the previous theorem to complete the proof of Corollary 1.6.

Proof of Corollary 1.6 Properties (1) and (2) are equivalent by Corollary 1.3.

If $H$ is undistorted in $G$, then it is relatively quasiconvex by Theorem 1.5. Recall that since $G$ is a geometrically finite Kleinian group its maximal parabolic subgroups $P$ are finitely generated and virtually abelian. Every subgroup $O$ of such a group $P$ is finitely generated and undistorted. If $H$ is relatively quasiconvex in $G$ it follows from Theorem 10.5 that $H$ is undistorted in $G$. Thus (2) and (3) are equivalent.

A geometrically finite Kleinian group $G$ acts properly discontinuously, cocompactly and isometrically on a CAT(0) space $Y$ with isolated flats (see, for instance, Hruska [22]). The author proves in [22] that a subgroup $H$ of a CAT(0) group $G$ with isolated flats is CAT( $(0)$-quasiconvex if and only if $H$ is finitely generated and undistorted in $G$. Thus (3) is equivalent to (4).

\section{References}

[1] I Agol, D Groves, J F Manning, Residual finiteness, QCERF and fillings of hyperbolic groups, Geom. Topol. 13 (2009) 1043-1073 MR2470970

[2] B N Apanasov, Geometrically finite hyperbolic structures on manifolds, Ann. Global Anal. Geom. 1 (1983) 1-22 MR739901

[3] A F Beardon, B Maskit, Limit points of Kleinian groups and finite sided fundamental polyhedra, Acta Math. 132 (1974) 1-12 MR0333164

[4] B H Bowditch, Geometrical finiteness for hyperbolic groups, J. Funct. Anal. 113 (1993) 245-317 MR1218098

[5] B H Bowditch, Geometrical finiteness with variable negative curvature, Duke Math. J. 77 (1995) 229-274 MR1317633

[6] B H Bowditch, A topological characterisation of hyperbolic groups, J. Amer. Math. Soc. 11 (1998) 643-667 MR1602069

[7] B H Bowditch, Relatively hyperbolic groups, Preprint, University of Southampton (1999) Available at http://eprints.soton.ac.uk/29769/1/bhb-relhyp.pdf

[8] S G Brick, On Dehn functions and products of groups, Trans. Amer. Math. Soc. 335 (1993) 369-384 MR1102884

[9] M R Bridson, A Haefliger, Metric spaces of non-positive curvature, Grund. der Math. Wissenschaften 319, Springer, Berlin (1999) MR1744486

[10] J W Cannon, D Cooper, A characterization of cocompact hyperbolic and finite-volume hyperbolic groups in dimension three, Trans. Amer. Math. Soc. 330 (1992) 419-431 MR1036000 
[11] F Dahmani, Combination of convergence groups, Geom. Topol. 7 (2003) 933-963 MR2026551

[12] F Dahmani, Les groupes relativement hyperboliques et leurs bords, Thèse, l'Université Louis Pasteur (Strasbourg I), Prépublication de l'Institut de Recherche Mathématique Avancée, 2003/13 (2003) MR2105643 Available at http:// www-irma.u-strasbg.fr/irma/publications/index.shtml

[13] C Druț, M Sapir, Tree-graded spaces and asymptotic cones of groups, Topology 44 (2005) 959-1058 MR2153979 With an appendix by D Osin and Sapir

[14] V Efromovich, On proximity geometry of Riemannian manifolds, Amer. Math. Soc. Transl. (2) 39 (1964) 167-170

[15] B Farb, Relatively hyperbolic groups, Geom. Funct. Anal. 8 (1998) 810-840 MR1650094

[16] E M Freden, Negatively curved groups have the convergence property. I, Ann. Acad. Sci. Fenn. Ser. A I Math. 20 (1995) 333-348 MR1346817

[17] H Garland, MS Raghunathan, Fundamental domains for lattices in ( $\mathbb{R})$-rank 1 semisimple Lie groups, Ann. of Math. (2) 92 (1970) 279-326 MR0267041

[18] F W Gehring, G J Martin, Discrete quasiconformal groups. I, Proc. London Math. Soc. (3) 55 (1987) 331-358 MR896224

[19] M Gromov, Hyperbolic groups, from: "Essays in group theory", (S M Gersten, editor), Math. Sci. Res. Inst. Publ. 8, Springer, New York (1987) 75-263 MR919829

[20] D Groves, J F Manning, Dehn filling in relatively hyperbolic groups, Israel J. Math. 168 (2008) 317-429 MR2448064

[21] V S Guba, M V Sapir, On Dehn functions of free products of groups, Proc. Amer. Math. Soc. 127 (1999) 1885-1891 MR1469408

[22] G C Hruska, Geometric invariants of spaces with isolated flats, Topology 44 (2005) 441-458 MR2114956

[23] A Lubotzky, Lattices in rank one Lie groups over local fields, Geom. Funct. Anal. 1 (1991) 406-431 MR1132296

[24] J F Manning, E Martínez-Pedroza, Separation of relatively quasiconvex subgroups, Pacific J. Math. 244 (2010) 309-334 MR2587434

[25] E Martínez-Pedroza, A note on quasiconvexity and relative hyperbolic structures arXiv:0811.2384

[26] E Martínez-Pedroza, Combination of quasiconvex subgroups of relatively hyperbolic groups, Groups Geom. Dyn. 3 (2009) 317-342 MR2486802

[27] D V Osin, Relatively hyperbolic groups: intrinsic geometry, algebraic properties, and algorithmic problems, Mem. Amer. Math. Soc. 179 (2006) vi+100 MR2182268 
[28] D V Osin, Peripheral fillings of relatively hyperbolic groups, Invent. Math. 167 (2007) 295-326 MR2270456

[29] D Y Rebbechi, Algorithmic properties of relatively hyperbolic groups, $\mathrm{PhD}$ thesis, Rutgers University (2001) arXiv:math.GR/0302245

[30] H Short, Quasiconvexity and a theorem of Howson's, from: "Group theory from a geometrical viewpoint (Trieste, 1990)", (É Ghys, A Haefliger, editors), World Sci. Publ., River Edge, NJ (1991) 168-176 MR1170365

[31] P Susskind, G A Swarup, Limit sets of geometrically finite hyperbolic groups, Amer. J. Math. 114 (1992) 233-250 MR1156565

[32] W P Thurston, The geometry and topology of three-manifolds, Princeton Univ. Math. Dept. Lecture Notes (1979) Available at http://msri.org/publications/books/ gt $3 \mathrm{~m} /$

[33] W P Thurston, Three-dimensional geometry and topology. Vol. 1, (S Levy, editor), Princeton Math. Series 35, Princeton Univ. Press (1997) MR1435975

[34] P Tukia, Convergence groups and Gromov's metric hyperbolic spaces, New Zealand J. Math. 23 (1994) 157-187 MR1313451

[35] P Tukia, Conical limit points and uniform convergence groups, J. Reine Angew. Math. 501 (1998) 71-98 MR1637829

[36] A Yaman, A topological characterisation of relatively hyperbolic groups, J. Reine Angew. Math. 566 (2004) 41-89 MR2039323

Department of Mathematical Sciences, University of Wisconsin-Milwaukee PO Box 413, Milwaukee, WI 53201, USA

chruska@uwm.edu

http://www. uwm.edu/ chruska

Received: 16 April 2009 Revised: 24 April 2010 